\title{
Constrained optimization of anti-symmetric cold-formed steel
}

\section{beam-column sections}

Hossein Parastesh ${ }^{1}$, Seyed Mohammad Mojtabaei ${ }^{2 *}$, Hamed Taji ${ }^{3}$, Iman Hajirasouliha ${ }^{1,2}$, Alireza Bagheri Sabbagh ${ }^{4}$

${ }^{1}$ Department of Civil Engineering, University of Science and Culture, Tehran, Iran

${ }^{2}$ Department of Civil and Structural Engineering, The University of Sheffield, Sheffield, UK

\author{
${ }^{3}$ Department of Civil Engineering, Technical and Vocational University (Khorasan Razavi Branch), Torbat Heydarieh, \\ Iran \\ ${ }^{4}$ School of Engineering, University of Aberdeen, Aberdeen, UK \\ * Corresponding author: smmojtabaei1@sheffield.ac.uk
}

\section{Abstract}

Flexibility in the manufacturing of cold-formed steel (CFS) cross-sectional shapes provides a unique opportunity to improve the load-carrying capacity of these elements, leading to more efficient and economic structural systems. This paper presents a practical constrained optimization methodology for pin-ended anti-symmetric CFS beam-columns members with different lengths subjected to various combinations of axial compression and bending moment. The optimization process is carried out using a Genetic Algorithm (GA) with respect to buckling resistance of CFS elements determined according to the Direct Strength Method (DSM). In total, 132 CFS beam-columns with three different lengths $(1000,2000$ and $3000 \mathrm{~mm}$ ) and eleven different cross-sections are optimized under concentrically compressive loads with different levels of eccentricities varying from 0 to $30 \mathrm{~mm}$. Each cross-section is formed using a certain number of fold-lines of steel plate, while the length and angle of the constituent elements of the cross-section are considered as the main design variables. To provide more practical beam-column elements, end-use constraints as well as a range of practical manufacturing and construction limitations are imposed on the selected cross-sections during the optimization process. A standard commercially available anti-symmetric $Z$ section is taken as a starting point of optimization and used to assess the efficiency of the optimized sections. The results show that, for the given plate width and thickness, the adopted optimization process can significantly increase the strength of beam-column members on average $62 \%, 92 \%$, and $188 \%$ for the short, medium and long length elements, respectively, compared to those with the standard section. It is also demonstrated that using more complex cross-sectional shapes does not necessarily provide higher strength for beam-column members. Finally, to verify the efficiency of the optimized sections, detailed nonlinear finite element models are developed using ABAQUS software. The developed models should prove useful for the efficient design of CFS beam-column elements in practice. 


\section{Keywords}

Cold-formed steel (CFS); Optimization; Genetic Algorithm; Beam-columns; Buckling modes; Finite Element (FE) model

\section{Introduction}

Cold-formed steel (CFS) structural elements are manufactured from thin steel plates using either pressbrake or rolling machine. CFS is capable of providing unique advantages such as lightweight, high strength-to-weight ratio, ease of handling and transportation, unrivalled construction speed, flexibility in forming of cross-sectional profiles and recyclability. Therefore, the use of CFS elements as the main loadbearing structural components is gaining more popularity in the modern construction industry. Under some load conditions, the CFS structural members can be subjected to combined axial compression and bending moment (known as beam-column elements). The strength and failure mode of CFS beam-column elements are highly dependent on the cross-section stress distribution generated by combined compression and bending actions [1-5]. In practical situations, the bending moment applied on the compression elements are resulted from (i): axial compressions with eccentricity (e.g. wall studs in ledger framing systems), (ii): combined axial compressions and end moments (e.g. moment-resisting frames), (iii): combined axial compressions and distributed transverse loads (e.g. CFS structures under lateral load actions). In the absence of either axial compression or bending moment, CFS elements behave as a pure beam or column, respectively. However, due to the existence of inevitable imperfections in framed structures, all CFS members generally act as beam-column elements.

Experimental and numerical studies previously investigated the structural behaviour of CFS beam-column elements subjected to various load combinations. An experimental program has been conducted by Torabian et al. $[1,2]$ to evaluate the buckling behaviour and failure mechanism of CFS beam-columns with $Z$ and lipped channel sections subjected to axial force and bi-axial moments. The results were then used to assess the reliability of the North American design standard (AISI-S100-12) [6], for predicting the strength of beam-columns, and to improve the current specification approach which utilizes a simple linear and conservative interaction equation. Experimental investigations were also conducted in other studies on cold-formed stainless steel beam-columns with Square Hollow Section (SHS) and Rectangular Hollow Section (RHS) $[3,4,7]$. It was demonstrated that while the codes' predictions are generally conservative, the European code [8] provides quite conservative predictions for the beam-column specimens compared to the American Specification [9] and Australian/New Zealand Standard [10] predictions.

Numerous research focused to improve the behaviour of CFS elements (i.e. beam and column) in terms of strength, stiffness, and energy dissipation by taking advantage of the high flexibility in their cross- 
sectional shape. This was achieved through optimization of the cross-sectional shapes for two different cases: (i) without any limitations on the general shape of the cross-section (i.e. unconstrained shape optimization) [11-17], (ii) with a predefined cross-sectional shape (i.e. constrained shape optimization) which is more practical and manufacturable [18-34]. Different optimization techniques have been used to achieve the optimum solutions for the CFS members including Genetic Algorithm (GA) [14, 17, 21, 27], Particle Swarm Optimization (PSO) [28, 31, 35], simulated annealing and gradient-based steepest descent method [12], Graph Theory and Ant Colony [15], Direct Multi-Search (DMS) [16], Hough Transform [30] and Big Bang-Big Crunch (BB-BC) [34]. It was reported by Ye et al. [28] that the flexural strength of CFS beam elements with standard commercially available sections can be significantly increased by only changing the dimensions of the cross-sections. Besides, Ye et al. carried out optimization process on a wide range of cross-sectional shapes with folded-flanges and edge and intermediate stiffeners [35] in order to further increase the flexural capacity of the CFS beam. Mojtabaei et al. [34] improved the stiffness and strength of CFS beam elements at serviceability and ultimate limit state conditions by using the BB-BC algorithm, respectively. CFS beam sections were also optimized for maximum energy dissipation to increase the seismic characteristics of the commercially available section and subsequently make them competence in seismic applications [32]. The results of unconstrained shape optimization on CFS columns [26] illustrated that the axial compressive strength can be considerably enhanced (up to $140 \%$ ) compared with the benchmark cross-section, while this enhancement reaches $49 \%$ for those with practical constraints [27]. Based on the results of constrained shape optimization on CFS column, Lee et al. [24] recommended optimum design curves for the various levels of axial load. In another study conducted by Ye et al. [31], the compressive capacity of the CFS lipped channel was optimized by considering the interactive local and flexural buckling modes. More recently, a coupled optimization framework at the element and structural levels was developed by Phan et al. [36] to find the optimum solutions for the CFS portal frames. They also conducted shape optimization to improve the flexural capacity of the CFS beam members used in bolted moment connections by taking into account the bimoment effects [37].

While CFS beam-column elements can be manufactured from simple $\mathrm{C}$ and $\mathrm{Z}$ sections to more complex shapes due to their great flexibility in forming process [38], a limited number of studies focused on the optimization of CFS beam-column members under different load combinations. In of the few available studies, Wang et al. [17] and Parastesh et al. [39] carried out optimization process on the CFS beamcolumn members with singly-symmetric open cross-sections to enhance their strength.

This study aims to provide a constrained optimization methodology for pin-ended anti-symmetric CFS beam-columns members subjected to various combinations of axial compression and bending moment. This can be cumbersome as the strength of a CFS member is controlled simultaneously by local, distortional and global buckling modes. The optimization process is carried out using the GA method with 
respect to buckling resistance of CFS elements determined according to the Direct Strength Method (DSM) [40]. To investigate the effect of different key design parameters, eleven cross-sections with different number of fold-lines (i.e. 4, 6, 8, 10, 12), three different element length (i.e. 1, 2, 3 m), and four different levels of load eccentricities (i.e. $0,10,20,30 \mathrm{~mm}$ ) are considered for the beam-column members. The length and angle of the constituent elements of the cross-sections (i.e. strips) are considered as main design variables during the optimization process. The capacities of optimized beamcolumn members are compared to those with the standard $Z$ sections to assess the efficiency of the proposed optimization methodology. Subsequently, detailed nonlinear Finite Element (FE) models are developed using ABAQUS software [41] to validate the calculated strength of the optimized sections and provide efficient tools for practical design of these sections.

\section{Constrained optimization procedure}

In this section, the optimization procedure of beam-column members is described in detail, including the cross-section design variables, end-use and manufacturing constraints, strength calculation according to DSM [40], optimization algorithm and definition of the optimization problem.

\subsection{Constituent strips of the cross-section}

Anti-symmetric cross-sections are generallv formed using different number of fold-lines during the manufacturing process. Fig. 1 shows a typical CFS anti-symmetric cross-section ( nsisting of six possible locations of the fold-lines. The cross-section was drawn on the XY plane and the origin of the coordinate system is placed on the free edge of the first strip (see Fig. 1). The axis of anti-symmetry was positioned at the mid-web height parallel to the $x$-axis. Since in this study the coil width and thickness of the steel plate is assumed to be constant, the capacity of CFS beam-column member mainly depends on the crosssectional shape. Therefore, the main design variables were considered to be the width of the strips $\left(l_{i}\right)$ and the angle of the strips $\left(\theta_{i}\right)$. It should be noted that $\theta_{i}$ is taken as the angle between the $i^{\text {th }}$ stip and the extension of $(i-1)^{\text {th }}$ stip except for $\theta_{1}$, which is the angle between the first strip and the $x$-axis as shown in Fig. 1. 


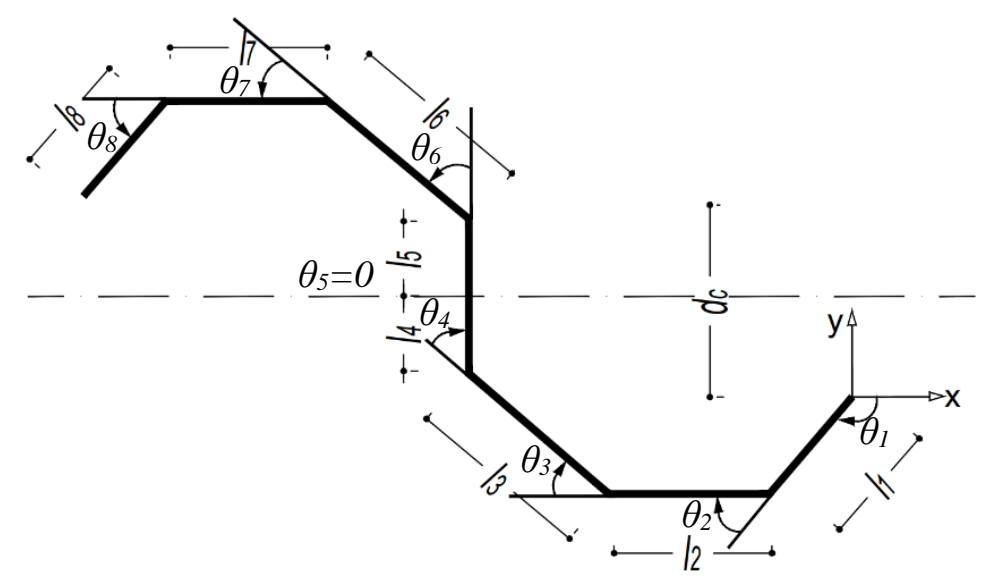

Fig. 1. Typical anti-symmetric cross-section with the selected design variables

128 The CFS cross-sections were characterized by the vectors $l$ and $\theta$, which indicate the lengths and angles of

129 the strips, respectively:

$130 \theta=\left[\theta_{1}, \theta_{2}, \ldots, \theta_{2 n}\right]$

$l=\left[l_{1}, l_{2}, \ldots, l_{2 n}\right]$

where $n$ is the number of constituent strips for half of the cross-section which is given by:

$133 n=n_{w}+n_{f}+n_{l}$

where $n_{l}, n_{f}$ and $n_{w}$ are the number of constituent strips of the lip, the flange and the web for half of the cross-section, respectively. By considering that one roller is required to form each of the non-zero angles (i.e. fold-lines), the relation between the number of rollers $\left(n_{\text {roll }}\right)$ and the total number of strips $(2 n)$ is as 137 follows:

$n_{\text {roll }}=2 n-2$

The total coil width of the steel plate $(W)$ is therefore defined by:

\subsection{Practical constraints and implementation}

To ensure the optimal cross-sections are feasible and practical, the following end-use and manufacturing constraints are imposed on the constituent strips $(l)$ and their angles $(\theta)$ :

a) Cross-sectional shape: The basic overall shape of all cross-sections are limited to anti-symmetric $Z$ shape section. Therefore, it is required to avoid overlapping of the cross-sectional strips. In this study, to simplify the optimization problem, the best design solutions are found for the design variables of half of 
the cross-sections. Considering that the sections are anti-symmetric, for the other half of the crosssection the length and angle vectors are given by [26]:

149

150

151

152

153

154

155

156

157

158

159

160

161

162

163

164

165

166

167

168

169

170

171

172

173

174

175

$l_{2 n-i+1}=l_{i+1}$

$\theta_{2 n-i+1}=-\theta_{i+1}$

where $i=1,2, \ldots, \frac{n_{\text {roll }}}{2}$

b) Utility pass-through: The distance between the free edges of the bottom and the upper lips in the ydirection ( $d c$ in Fig. 1) was required to be at least $25 \mathrm{~mm}$ to allow the building utilities to be positioned inside the roofing system as suggested by Leng et al. [15]. The central vertical strip (i.e. web) was also set to have a minimum height of $50 \mathrm{~mm}$ and restricted to be perpendicular to the flange in order to facilitate perforation and installation of the utilities. It should be noted that, at the point of symmetric (i.e. mid web), the angle between the connected strips was equal to zero $\left(\theta_{\mathrm{n}+1}=0\right) . \theta_{n}$ and $\theta_{\mathrm{n}+1}$ are both dependent design variables, while $\theta_{n}$ can be formulated by Eq. (8).

$\theta_{n}=-\frac{\pi}{2}-\sum_{n_{l}+n_{f}+1}^{n-1} \theta_{i}$

In the above equation, if $n<n_{l}+n_{f}+2$, the term $\sum_{n_{l}+n_{f}+1}^{n-1} \theta_{i}$ is taken to be zero.

c) Ease of connection: The flange strips of the cross-sections are considered to be always parallel to $x$ axis, and are taken to have a minimum width of $25 \mathrm{~mm}$. It should be noted that the bottom flange strip has lower $y$-coordinate than the other strips, in order to facilitate the attachment of deck and plywood boards to this member as suggested by Leng et al. [15]. In this study, the number of flange strips is always equal to one $\left(n_{f}=1\right)$. The flange angle is also a dependent variable which can be obtained using the following equation:

$\theta_{n_{l}+1}=-\pi-\sum_{i=1}^{n_{l}} \theta_{i}$

d) Strip width: To make feasible and practical cross-sections, a sufficient length for each strip is imposed on the cross-section. It was recommended by the industrial collaborator of this project to restrict the minimum length of strips to $10 \mathrm{~mm}$.

e) Rounded corner: The value of the radius-to-thickness ratio of the rounded corners is taken to be $3 t$ (where $t$ is the thickness of plate element) as suggested by AISI-S100-16 [40].

f) Manufacturing constraints: In this study, it is assumed that an even number of rollers between 4 and 12 are used to manufacture the anti-symmetric cross-sections. In practice, the maximum number of the lip strips $\left(n_{l, \max }\right)$ is taken based on the number of the available rollers. Therefore, by considering the Eqs. 
(4) and (5), $n_{l, \max }$ is equal to 1,2 and 3 when the number of half cross-section strips $(n)$ is 3,4 and 5 ,

177 respectively.

\subsection{Design of CFS members based on DSM}

179

180

181

182

183

184

185

186

187

188

189

190

191

192

193

194

195

196

197

$\left\{\begin{array}{cc}P_{n e}=\left(0.658^{\lambda_{c}^{2}}\right) P_{y} & \text { for } \lambda_{c} \leq 1.5 \\ P_{n e}=\left(\frac{0.877}{\lambda_{c}^{2}}\right) P_{y} & \text { for } \lambda_{c}>1.5\end{array}\right.$

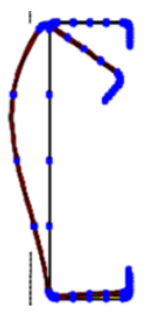

(b)

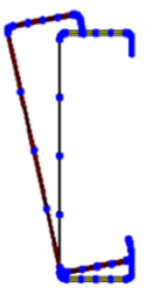

(c)

Fig. 2. Types of buckling modes: (a) local, (b) distortional, (c) global

\subsubsection{Buckling resistance of the member under axial compressive load}

Based on AISI [40], axial compressive resistance for global buckling is determined using compressive yield load $P_{y}=A_{g} f_{y}$ and slenderness ratio $\lambda_{c}=\sqrt{P_{y} / P_{c r e}}$ (where $A_{g}$ is the gros cross-sectional properties, $f_{y}$ is the yield stress, and $P_{c r e}$ denotes elastic gloal buckling stress):

AISI takes into account the local-global interaction mode through local-global slenderness ratio $\lambda_{l}=$ $\sqrt{P_{n e} / P_{c r l}}$, and therefore, the nominal axial resistance for local buckling is defined by [40]:

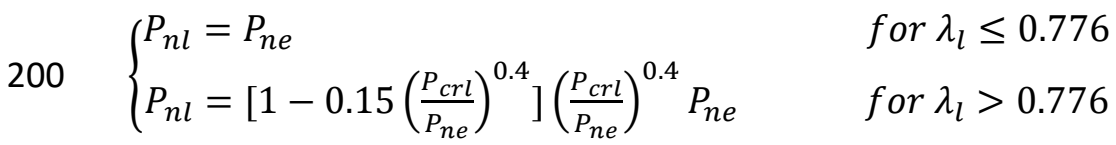


The nominal axial resistance for distortional buckling is expressed in terms of distortional buckling

202 slenderness ratio $\lambda_{d}=\sqrt{P_{y} / P_{c r d}}$ :

$203 \begin{cases}P_{n d}=P_{y} & \text { for } \lambda_{d} \leq 0.561 \\ P_{n d}=\left[1-0.25\left(\frac{P_{c r d}}{P_{y}}\right)^{0.6}\right]\left(\frac{P_{c r d}}{P_{y}}\right)^{0.6} P_{y} & \text { for } \lambda_{d}>0.561\end{cases}$

204 The nominal axial resistance of the compression member $\left(P_{n}\right)$ is then calculated by using the minimum

205 value of the resistances determined in Eqs. (10) to (12).

206

207

208

209

$$
\left\{\begin{array}{lr}
M_{n e}=M_{\text {cre }} & \text { for } M_{\text {cre }} \leq 0.56 M_{y} \\
M_{n e}=\frac{10}{9} M_{y}\left(1-\frac{10 M_{y}}{36 M_{\text {cre }}}\right) & \text { for } 2.78 M_{y}>M_{\text {cre }}>0.56 M_{y} \\
M_{n e}=M_{y} & \text { for } M_{\text {cre }} \geq 2.78 M_{y}
\end{array}\right.
$$

\subsubsection{Buckling resistance of the member under bending}

The nominal flexural resistance is determined in terms of the flexural yield moment $M_{y}=W_{y} f_{y}$ and the elastic critical lateral-torsional $M_{\text {cre }}$ (where $W_{y}$ is the elastic section modulus):

The nominal flexural resistance for local buckling considering local-global interaction is also expressed as a function of local-global slenderness ratio $\lambda_{l}=\sqrt{M_{n e} / M_{c r l}}$ :

$$
\begin{cases}M_{n l}=M_{n e} & \text { for } \lambda_{l} \leq 0.776 \\ M_{n l}=\left[1-0.15\left(\frac{M_{c r l}}{M_{n e}}\right)^{0.4}\right]\left(\frac{M_{c r l}}{M_{n e}}\right)^{0.4} M_{n e} & \text { for } \lambda_{l}>0.776\end{cases}
$$

213 The nominal flexural resistance for distortional buckling is determined using distortional buckling slenderness ratio $\lambda_{d}=\sqrt{M_{y} / M_{c r d}}$ :

$$
\begin{cases}M_{n d}=M_{y} & \text { for } \lambda_{d} \leq 0.673 \\ M_{n d}=\left[1-0.22\left(\frac{M_{c r d}}{M_{y}}\right)^{0.5}\right]\left(\frac{M_{c r d}}{M_{y}}\right)^{0.5} M_{y} & \text { for } \lambda_{d}>0.673\end{cases}
$$

The nominal flexural resistance of the CFS member $\left(M_{n}\right)$ is then obtained from the minimum value of the resistances determined in Eqs. (13) to (15).

\subsubsection{Buckling resistance of the beam-column member under combined axial compressive load} and bending

AISI recommends two linear interaction equations for the CFS members under combined axial

222

$$
\frac{P}{P_{n}}+\frac{C_{m x} M_{x}}{M_{n x} \alpha_{x}}+\frac{C_{m y} M_{y}}{M_{n y} \alpha_{y}} \leq 1.0
$$


In the above equations $P, M_{x}$ and $M_{y}$, are defined as the applied axial compression load and bending moments about the $\mathrm{x}$ and $\mathrm{y}$-axes, respectively. $C_{M}=0.6-0.4\left(\frac{M_{1}}{M_{2}}\right)$ is the moment gradient factor about x- or $y$-axis, where $M_{1}$ and $M_{2}$ are the ratio of the smaller to the larger moment at the two ends of the member. $\alpha$ is the moment amplification factor (about $\mathrm{x}$ - or $\mathrm{y}$-axis) determined by :

$\alpha=1-\frac{P}{P_{E}}$

where $P_{E}=\frac{\pi^{2} E I}{(K L)^{2}}$ is the elastic buckling load.

\subsection{Implementation of the GA}

In this study, a Genetic Algorithm (GA) is used to find the best solution for the optimization problem. GA was initially developed according to the principles of natural evolution, where potential solutions are evolved using certain selection rules to find the optimal answer for an optimization target (i.e. fitness function) $[43,44]$. To this end, an initial population of chromosomes is first generated, in which each chromosome represents a potential candidate to the problem. The generated chromosomes are then assessed according to the optimization target, and the best candidates (i.e. with greater fitness function values) possess a higher chance of reproduction. The formulation of the chromosome assessment plays an important role to obtain faster convergence and consequently reduce computational cost. In this study, the objective function is subjected to linear inequality constraints and bounds. Therefore, the optimization constraints can be satisfied at every generation by randomly selecting the input design parameters (i.e. constituent strips and their angles) within their acceptable range. A summary of the Genetic Algorithm used in this study is given in Fig. 3, where feasible cross-sections were generated by implementing the selected design constraints

In this study, the population size and the number of generations were selected equal to 80 and 100 , respectively. The criterion for terminating the program is a predefined total number of generations. Crossover rate of $r_{c}=0.8$ and mutation rate of $r_{m}=0.02$ were taken in the optimization process. The "roulette wheel" method was used to select parents, while a single point crossover was employed to swap variable design vectors between two parents. 


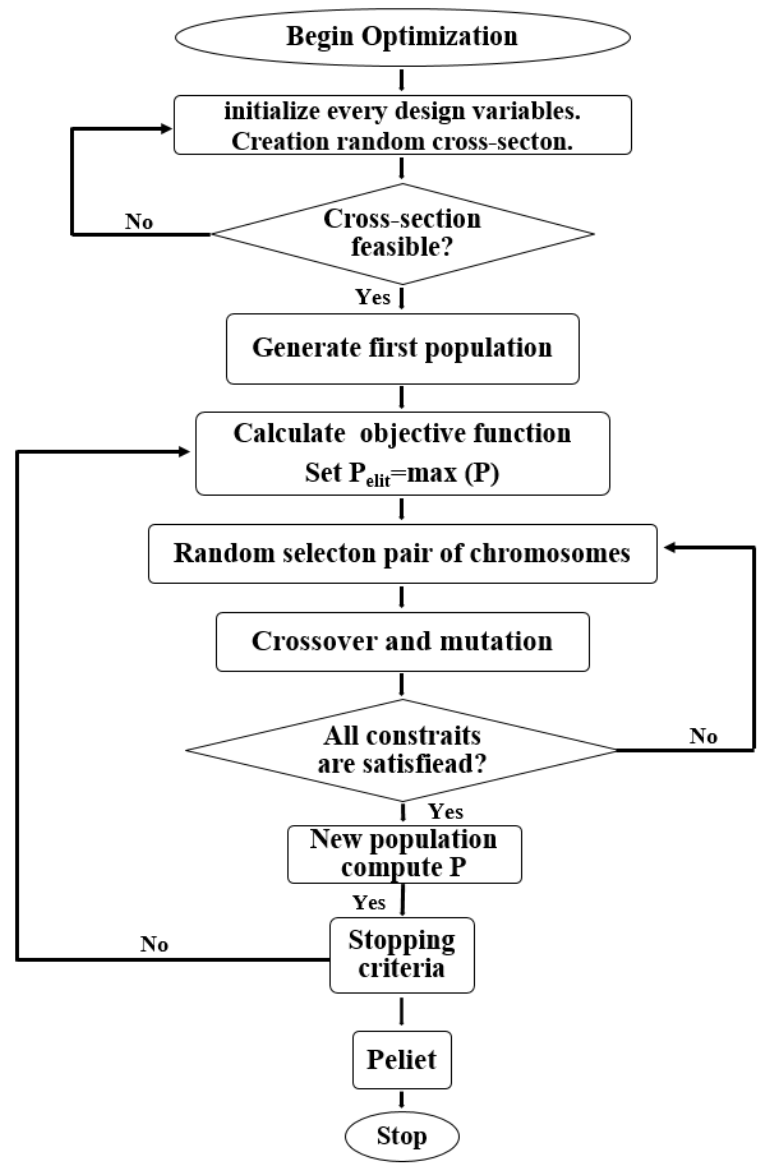

Fig. 3. Flowchart of the optimization process using GA

\subsection{Problem definition}

The main objective of the optimization process is to find the best design solutions for the strength of the pin-ended CFS beam-column members with anti-symmetric cross-sectional shapes under various combinations of axial compressive load and bending moment. To take into account the effect of various buckling modes in the optimization process, eleven different cross-sectional shapes and three different element lengths (1000, 2000 and $3000 \mathrm{~mm}$ ) were selected in this study. The number of rollers and lip strips were considered as the key independent design parameters for the cross-sectional shape. Hence, the selected cross-sections were formed using a different number of rollers ( $n_{\text {roll }}=4,6,8,10$ and 12) and lip strips $\left(n_{l}=1,2\right.$, and 3$)$, while the number of strips for web and flange were taken equal to $n_{w}=$ $1, n_{f}=1$, respectively. Table 1 lists the selected cross-sections identified with two numbers, where the first value stands for the number of rollers $\left(n_{\text {roll }}\right)$ and the second one indicates the number of lip strips $\left(n_{l}\right)$. A standard commercially available z-shape section, as shown in Fig 4 , was used as a benchmark to evaluate the efficiency of the optimization results. The total coil width and the thickness of the steel plate were selected to be $W=320 \mathrm{~mm}$ and $t=1 \mathrm{~mm}$, respectively. The elastic modulus, the Poisson's ratio, and the yield stress of the CFS material were taken as $E=210 \mathrm{GPa}, v=0.3$, and $f_{y}=350 \mathrm{MPa}$, respectively. 
The interactive compression-bending strength of CFS members was determined according to DSM [40]. In this study, the interaction of axial compression and bending was provided by applying eccentric compressive load $(P)$ with different levels of eccentricity $(e)$ varying from 0 to $30 \mathrm{~mm}$ with $10 \mathrm{~mm}$ intervals. It was assumed that the excentricity was placed on the minor axis (y), and therefore, the major axis bending moment was generated about $\mathrm{x}$-axis $\left(M_{x}\right)$, as shown in Fig. 4.

272 Substituting $M_{x}=P . e$ into Eqs. (16) and (17) and rearranging the equations yields:

273

$\left(\frac{-M_{n x}}{P_{E}}\right) \cdot P^{2}+\left(M_{n x}+P_{n} C_{m x} e+\frac{P_{n} M_{n x}}{P_{E}}\right) P-P_{n} \cdot M_{n x}=0$

$P-\frac{P_{n} M_{n x}}{M_{n x}+P_{n} e}=0$

where the lower value of $P$ is taken as the capacity of the beam-column member. Therefore, the optimization fitness function, which is the function of the width and angle of the cross-section strips, is given by:

279 where $P(l, \theta)$ is the minimum of Eqs. (19) and (20).

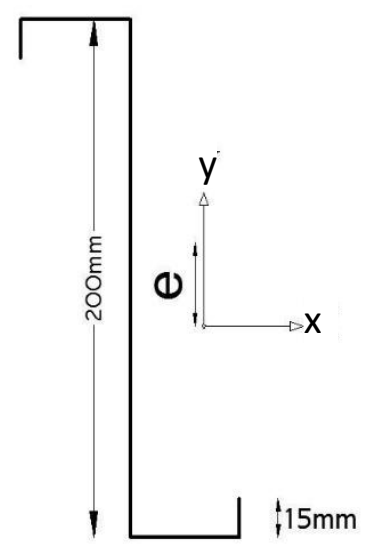

In this study, the optimization procedure was carried out using the following steps:

1) The number of rollers $\left(n_{\text {roll }}\right)$, lip strips $\left(n_{l}\right)$ and flange strips $\left(n_{f}\right)$ were first specified for the half crosssection. The number of web strips $\left(n_{w}\right)$ and the total number of strips in the whole cross-section $(2 n)$ are then calculated by using Eqs. (4), (5).

2) The adopted optimization algorithm (GA) randomly generated the half cross-section using the two vectors of design variables, including length $(l)$ and angle $(\theta)$, by considering the imposed design constraints specified in Section 2.2. 
3) The GA was linked to CUFSM software [42] to determine the elastic buckling resistance of CFS element with the generated cross-section. It should be noted that the calculation of elastic buckling loads is not

291

292

293

294

295 straight forward using Finite Strip Method when no local minimum exists, especially for the irregular cross-sectional shapes. Since Finite Strip analysis normally leads to one or no local minimum, it is not capable of identifying the local/distortional buckling stresses. Therefore, the constrained Finite Strip Method has been adopted in this study to automatically obtain pure buckling modes (i.e. local and distortional) $[45,46]$. For such cases, the method proposed by Gilbert et al. [13] can be also used to estimate elastic buckling loads during the optimization process.

4) The DSM [40] was adopted to calculate the axial compression capacity $\left(P_{n}\right)$ and the flexural strength $\left(M_{\mathrm{nx}}\right)$ of the selected beam-column element.

5) The buckling capacity of the beam-column member $(P)$ was calculated using either Eqs. 19 and 20, whichever results in a lower value. Subsequently, this loop was continued until convergence (or the maximum number of generations) was achieved.

\section{Optimization results and discussions}

The optimization process was carried out on the cross-sections discussed in section 2.5 by developing two distinct MATLAB codes [47] for the DSM calculations and the GA optimization. The purpose of the optimization process was to find the optimum shapes of the selected cross-sections, which were defined in terms of the lengths and angles of the cross-sectional constituent elements (i.e. strips). Each crosssection was optimized five times to guarantee that the best solution has been achieved. The results demonstrated that the differences between the results of the five runs were negligible (less than $1 \%$ ). During the process of optimization, the convergence was obtained after approximately 50 generations for all selected cross-sections. As an example, the iteration history of the optimization process for $1000 \mathrm{~mm}$ beam-column members with $10-1$ cross-section and $e=0$ is shown in Fig. 5, where the convergence is achieved after 47 iterations.

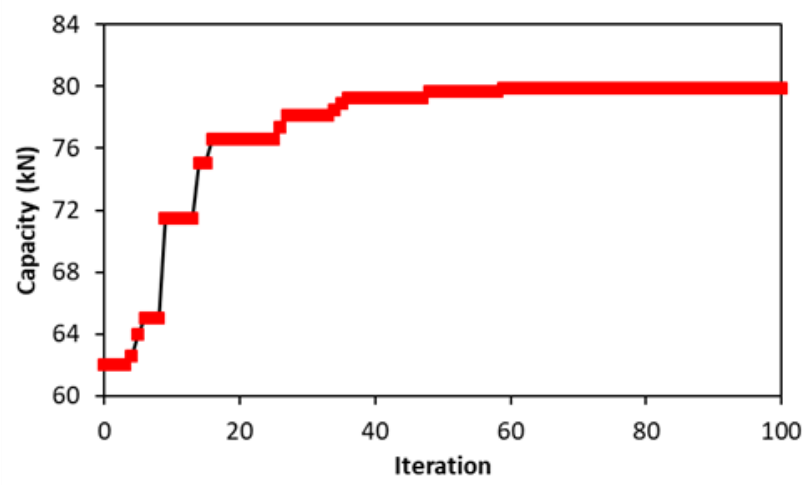


Tables 1 and 2 show the optimal shape of eleven different cross-sections that resulted in the best design solutions for the beam-column members with short, medium and long length (i.e. 1000, 2000 and $3000 \mathrm{~mm}$, respectively) under different load combinations (i.e. different eccentricity levels). As can be seen, all the imposed practical constraints have been satisfied in the obtained optimum cross-sections. Besides, it is shown that for the given number of foldings and lip strips, the optimum cross-sectional shapes were affected by the element length and value of eccentricity. For the members with the short length, a meaningful trend can be seen for the optimum shapes of the sections, in which the cross-section tend to have a more spread shape when the eccentricity increases. This is especially evident for the 6-1, 6-2, 8-1, 10-2 and 12-2 cross-sections. It can be noted that for the medium and long length members, the general shape of the optimum solutions was less affected by increasing the eccentricity. This can be attributed to the dominant behaviour of the global buckling modes for the longer elements.

Tables A1 to A3 in the appendix list the calculated web heigh of the standard $Z$ and optimum sections $(h)$ as well as their cross-sectional properties including moments of inertia about $\mathrm{x}$ - and $\mathrm{y}$-axes $\left(I_{x}, I_{y}\right)$ and principal axes $\left(I_{1}, I_{2}\right)$, and warping coefficient $\left(C_{w}\right)$. It should be noted that the AISI [40] stipulates to employ the principal moment of inertia through the calculations of global buckling resistance. These tables also show the nominal buckling resistances of the elements for pure compression $\left(P_{n}\right)$ and pure bending moment $\left(M_{n x}\right)$ along with their corresponding dominant buckling modes, and the strength of the beam-columns $(P)$ for the predefined eccentricity levels (e). In the tables, the letters $L, D$, and L-G denote local, distorional and local-global buckling modes obtained from elastic buckling analysis using CUFSM [42]. As expected, while cross-sectional instabilities (i.e. local, distortional) were dominant for the short members, the global buckling mode governed the design when the ratio of the member length to the cross-sectional height $(L / h)$ is increased. In addition, the possibility of local and distortional buckling was increased by increasing the length of the lip strips (i.e. the width-to-thickness ratio of the plates) and the number of lip strips, respectively.

The results of strength ratios of the beam-column members with different optimized cross-sections over those with the standard section $\left(P / P_{\text {stan }}\right)$ for $1000 \mathrm{~mm}, 2000 \mathrm{~mm}$ and $3000 \mathrm{~mm}$ element lengths are shown in Figs. 6, 7 and 8, respectively. It can be seen that increasing the number of lip strips can generally improve the optimized results for long beam-columns, while this is not necessarily the case for the short and medium length elements. This implies that increasing the number of lips in the optimum sections increases the global buckling resistance, which governs the results in the long length elements. Besides for the case of short and medium length elements, since each optimized beam-column fails in a different buckling mode (see Tables $A 1, A 2$, and $A 3$ ), a general trend cannot be seen for the results of $\left(P / P_{\text {stan }}\right)$ in Figs. 6 and 7. 
Table 1. Optimized cross-sections for $1000 \mathrm{~mm}$ long beam-columns

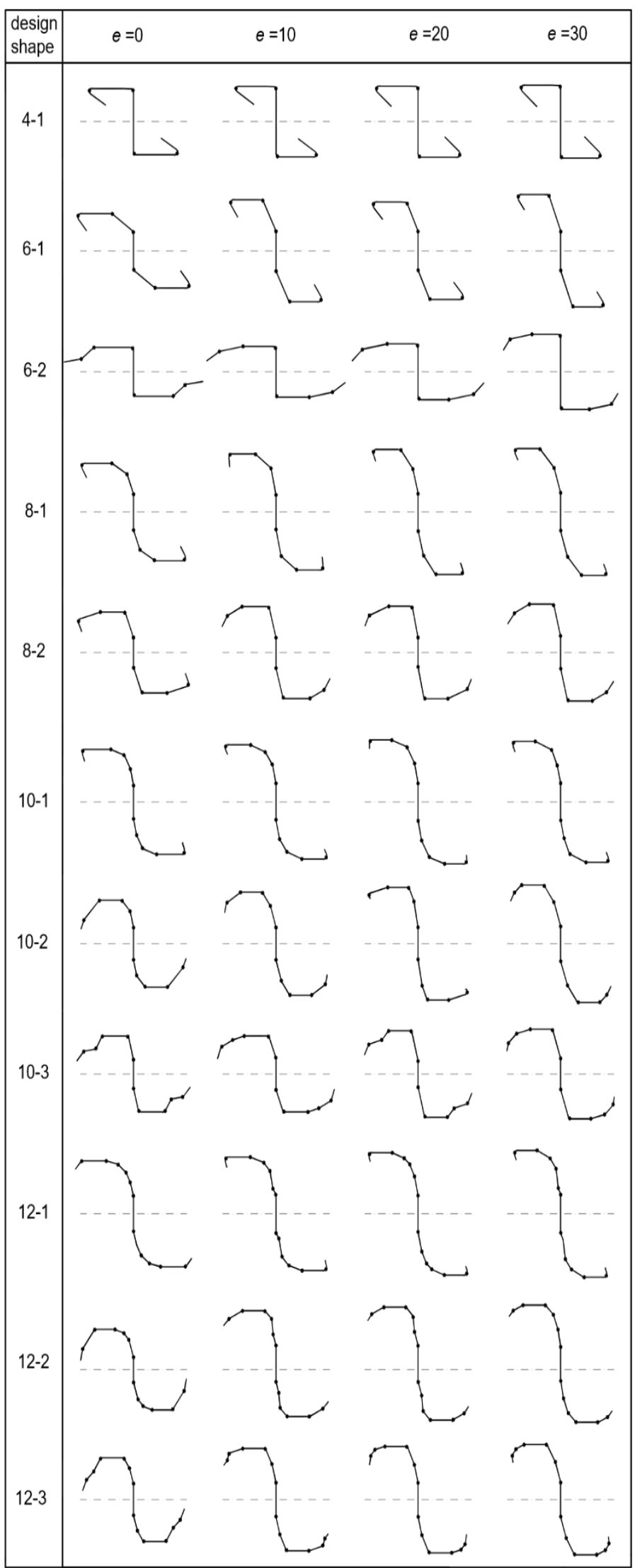


Table 2. Optimized cross-sections for $2000 \mathrm{~mm}$ and $3000 \mathrm{~mm}$ long beam-columns.

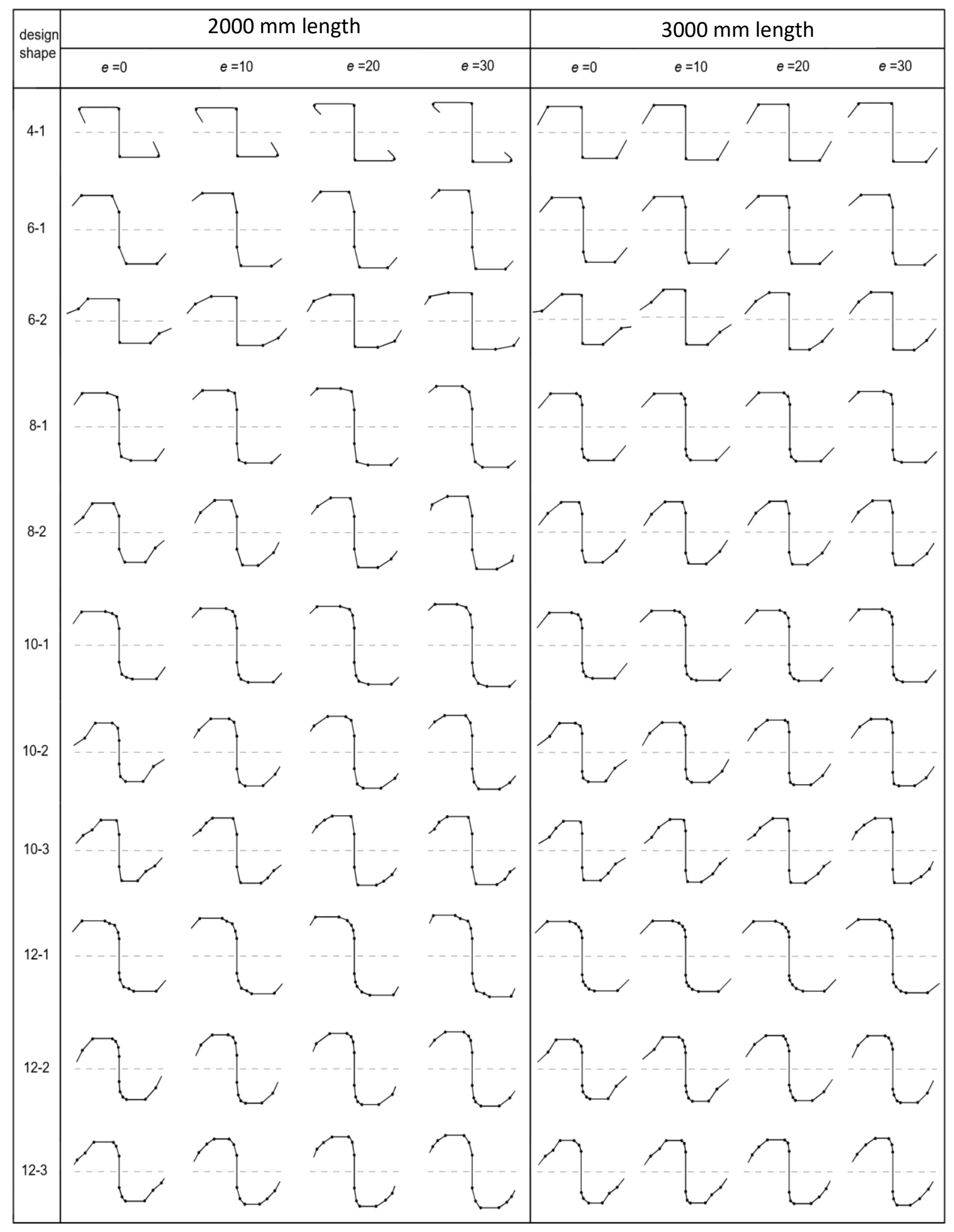


For better comparison, the capacities of the optimized beam-column elements to the standard $Z$ section for different levels of load eccentricity are presented in Fig. 9, based on the average of the results for each member length. It can be seen that, on average, the eccentricity of the load did not considerably influence the efficiency of the optimization process. The results also show that, for the given plate width and thickness, the adopted optimization process can significantly increase the strength of beam-column members $(P)$ on average $62 \%, 92 \%$, and $188 \%$ for the short (i.e. $L=1000 \mathrm{~mm}$ ), medium (i.e. $L=$ $2000 \mathrm{~mm}$ ) and long (i.e. $L=3000 \mathrm{~mm}$ ) length elements compared to those with standard $Z$ section, respectively. This implies that, in general, increasing the length of the beam-column members leads to a noticeable increase in the efficiency of the optimization. A similar conclusion has been previously reported by Ye et al. [31] for $C$ channel columns under pure axial compression load. For the members with long length, since the optimized elements experienced an approximately similar type of instability (i.e. global buckling), the strength of the optimized members followed a general trend, where increasing the level of eccentricity could always result in a reduction in the efficiency of the optimization.

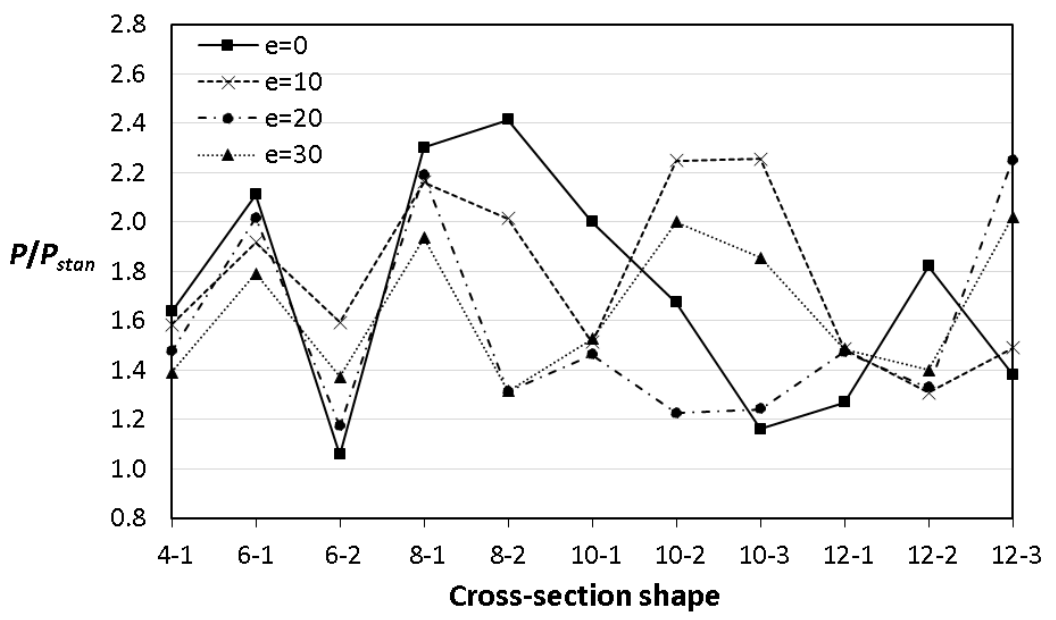

Fig. 6. Strength ratios of the $1000 \mathrm{~mm}$ beam-column members with different optimized cross-sections over the standard section

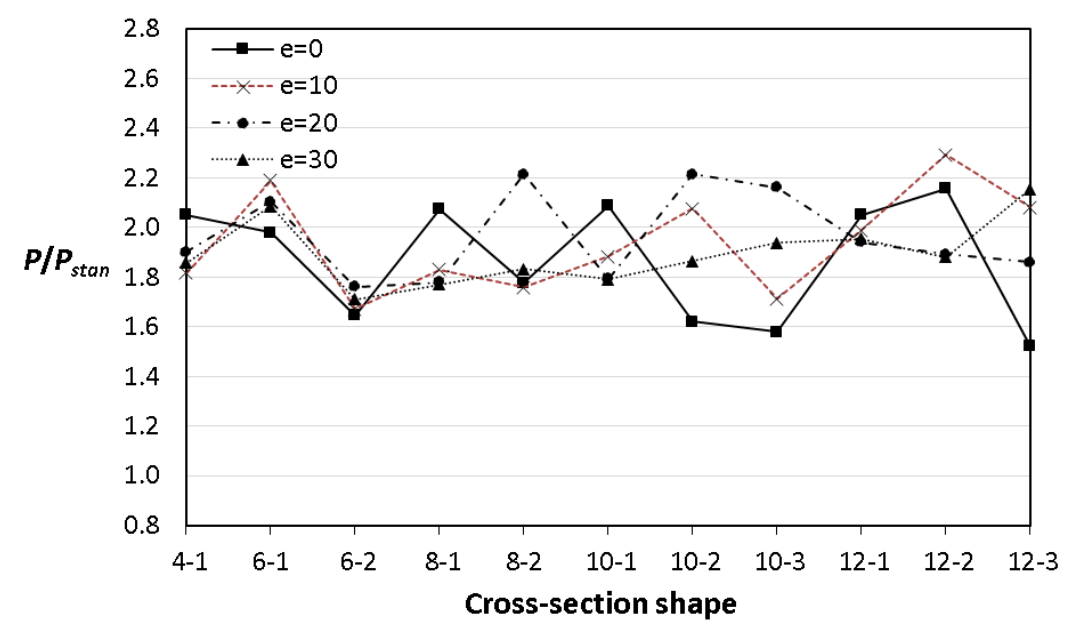

Fig. 7. Strength ratios of the $2000 \mathrm{~mm}$ beam-column members with different optimized cross-sections over the standard section 


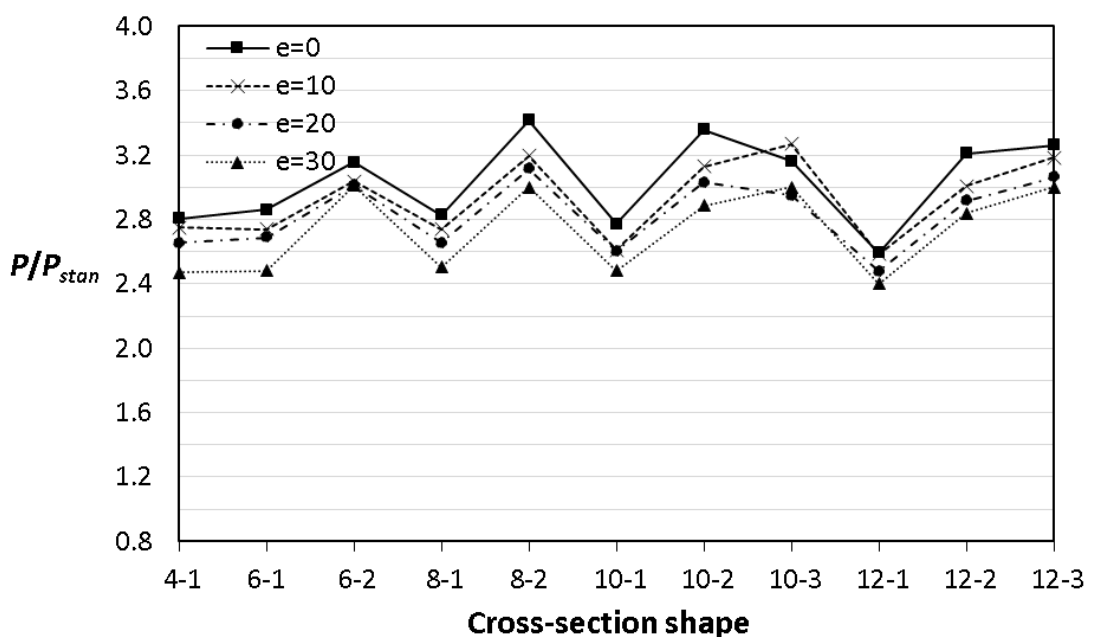

Fig. 8. Strength ratios of the $3000 \mathrm{~mm}$ beam-column members with different optimized cross-sections over the standard section

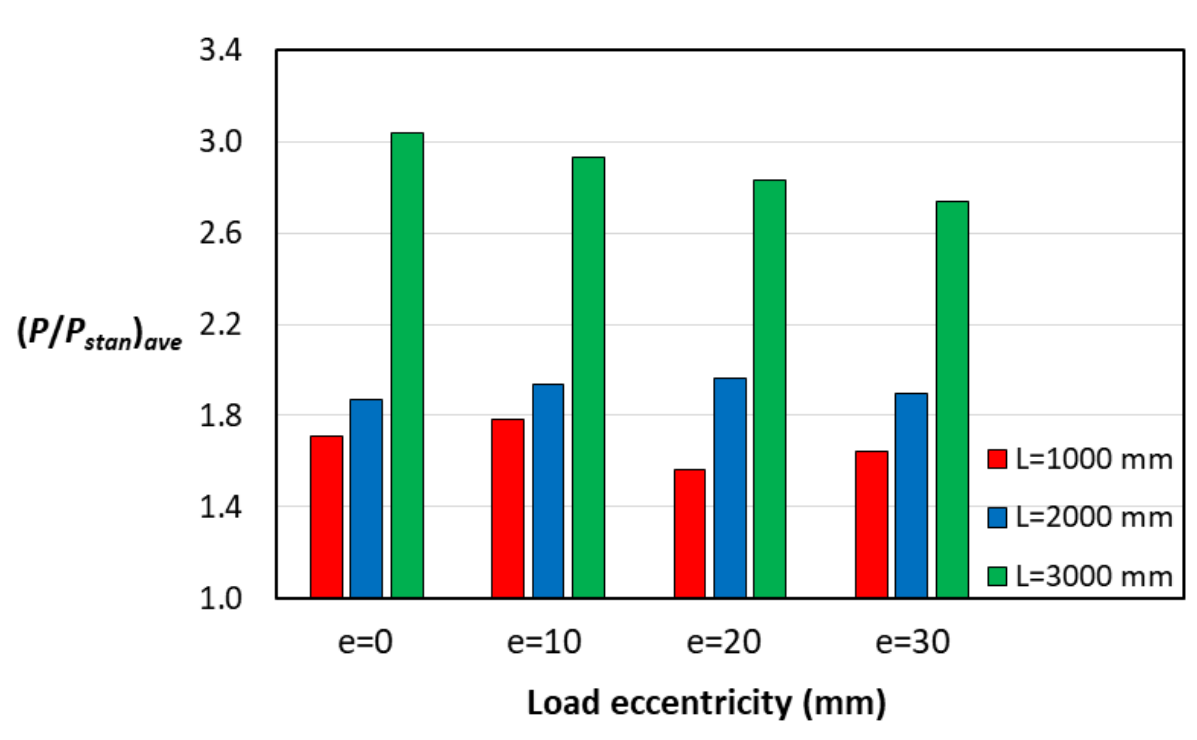

Fig. 9. Average capacities of the optimized beam-column elements to the standard $Z$ section under different load eccentricities and with 1000, 2000 and $3000 \mathrm{~mm}$ length

The variations of the web height of the optimized cross-sections $(h)$ and standard $Z$ section $\left(h_{\text {stan }}=\right.$ $200 \mathrm{~mm}$ ) are shown in Figs. 10, 11 and 12 for the members with different length. The ratio of $h / h_{\text {stan }}$ is varying from 0.35 to $0.9,0.42$ to 0.77 , and 0.47 to 0.69 for the beam-column members with 1000, 2000 and $3000 \mathrm{~mm}$ length, respectively. This indicates that the optimum cross-sectional shapes of the short members (i.e. $L=1000 \mathrm{~mm}$ ) varied more significantly compared to the other lengths by changing the imposed practical constraints (i.e. number of rollers and lip strips) and the applied eccentricity $(e)$. This can be attributed to the fact that for the short elements different cross-sectional instabilities can govern the design. Figs. 10, 11 and 12 also demonstrate that, in general, the height of the beam-column crosssections increased by increasing the level of eccentricity. This is referred to the fact that increasing the eccentricity leads to a higher value of major axis bending moment (i.e. x-axis), hence the optimization 
393

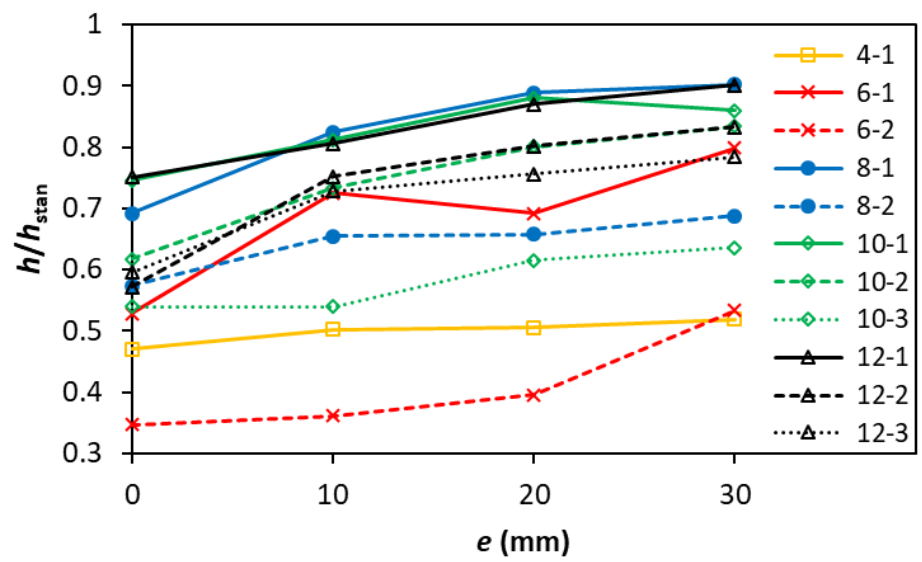

Fig. 10. Comparison between the web heights of standard $Z$ and optimized cross-sections for the beamcolumns with $1000 \mathrm{~mm}$ length

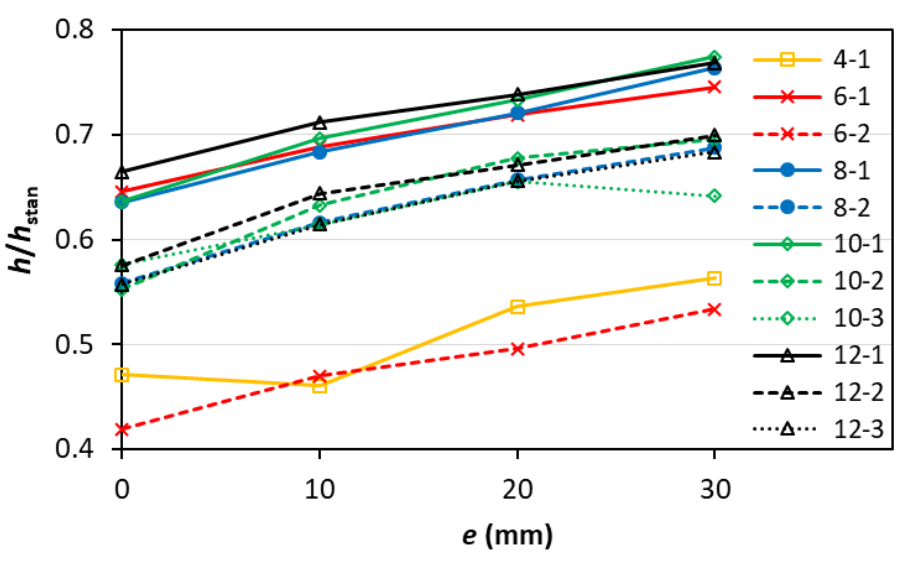

Fig. 11. Comparison between the web heights of standard $Z$ and optimized cross-sections for the beamcolumns with $2000 \mathrm{~mm}$ length

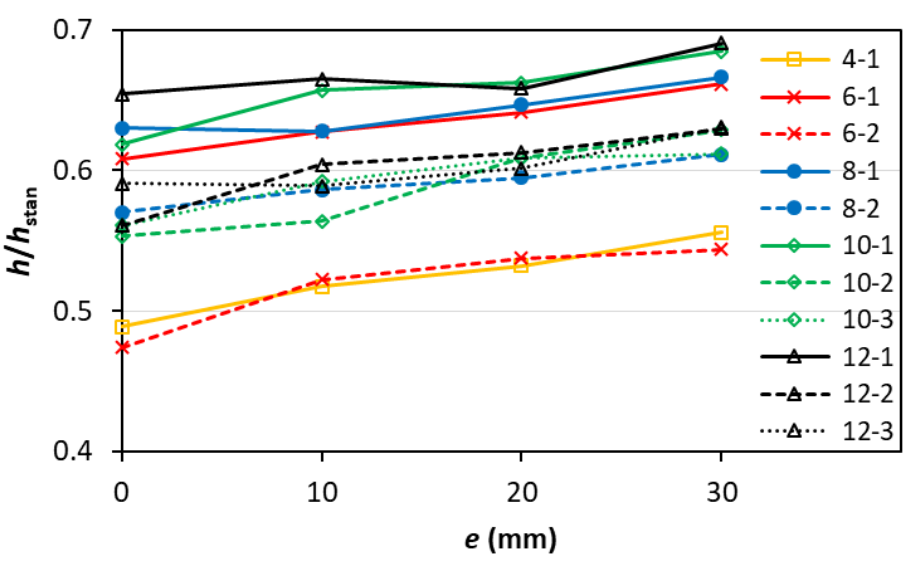

Fig. 12. Comparison between the web heights of standard $Z$ and optimized cross-sections for the beam-columns with $3000 \mathrm{~mm}$ length

The relationships between the warping constants of the optimized cross-sections $C_{w}$ and the standard $Z$ section $C_{w, \text { stan }}$ are illustrated in Figs. 13, 14 and 15 for the members with 1000, 2000, and $3000 \mathrm{~mm}$ 
length, respectively. The results demonstrate that the warping constants of the majority of the optimized sections are noticeably increased (by up to $108 \%$ ) compared to the standard section. It is also shown that the ratio of $C_{w} / C_{w, \text { stan }}$ significantly fluctuated for the members with the short length (see Fig. 13), however, this ratio can be approximately considered to be constant for the long length elements $\left(C_{w} / C_{w, \text { stan }}=1.8\right)$. This implies that the optimized cross-sections for the short beam-column members (i.e. $L=1000 \mathrm{~mm}$ ) experienced different buckling modes, and therefore, their optimum shapes and consequently warping constants were considerably different with those obtained for the medium and long length elements (see Tables 1 and 2).

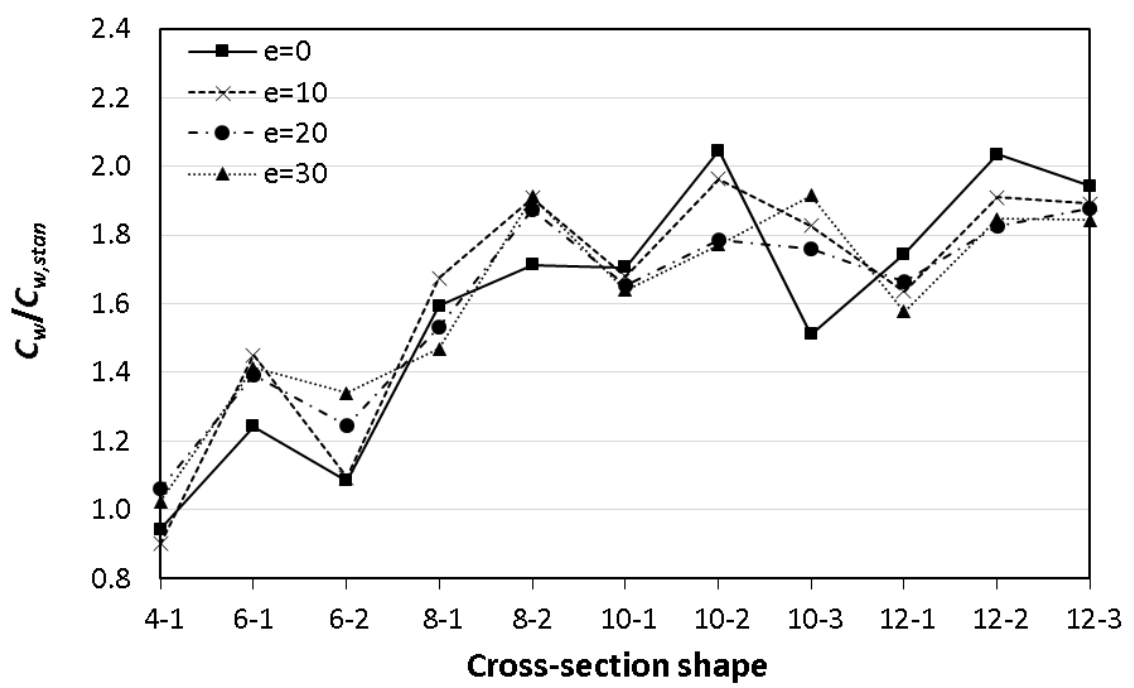

Fig. 13. Comparison between the warping constants of standard $Z$ and optimized cross-sections for the beam-columns with $1000 \mathrm{~mm}$ length

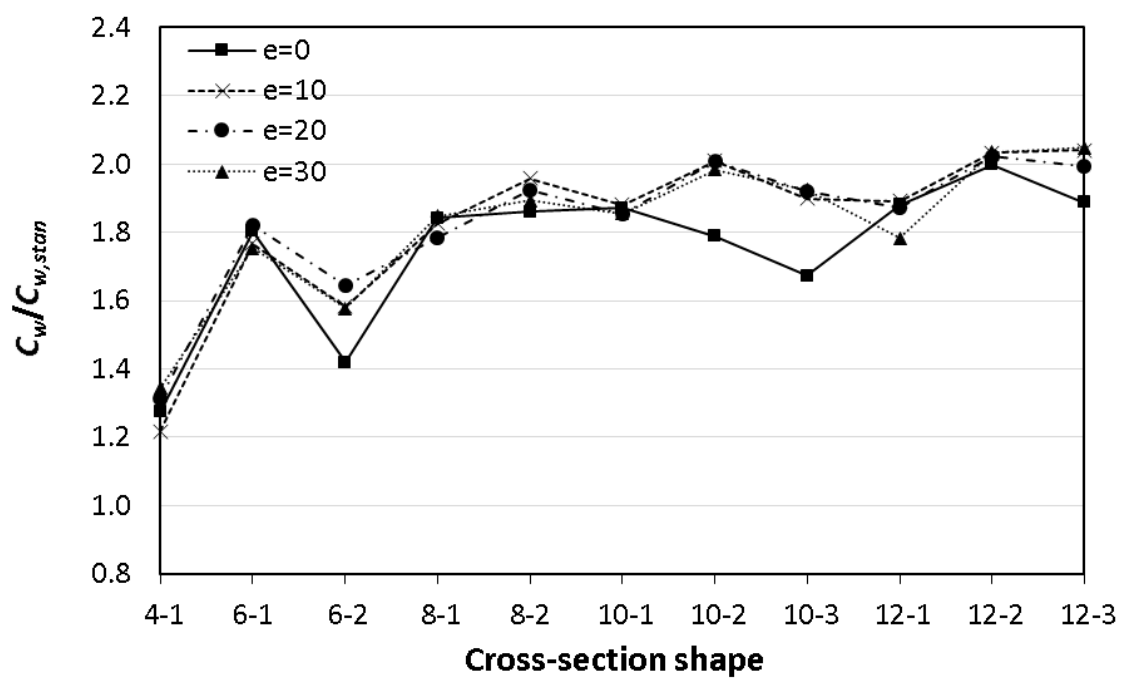

Fig. 14. Comparison between the warping constants of standard $Z$ and optimized cross-sections for the beam-columns with $2000 \mathrm{~mm}$ length 


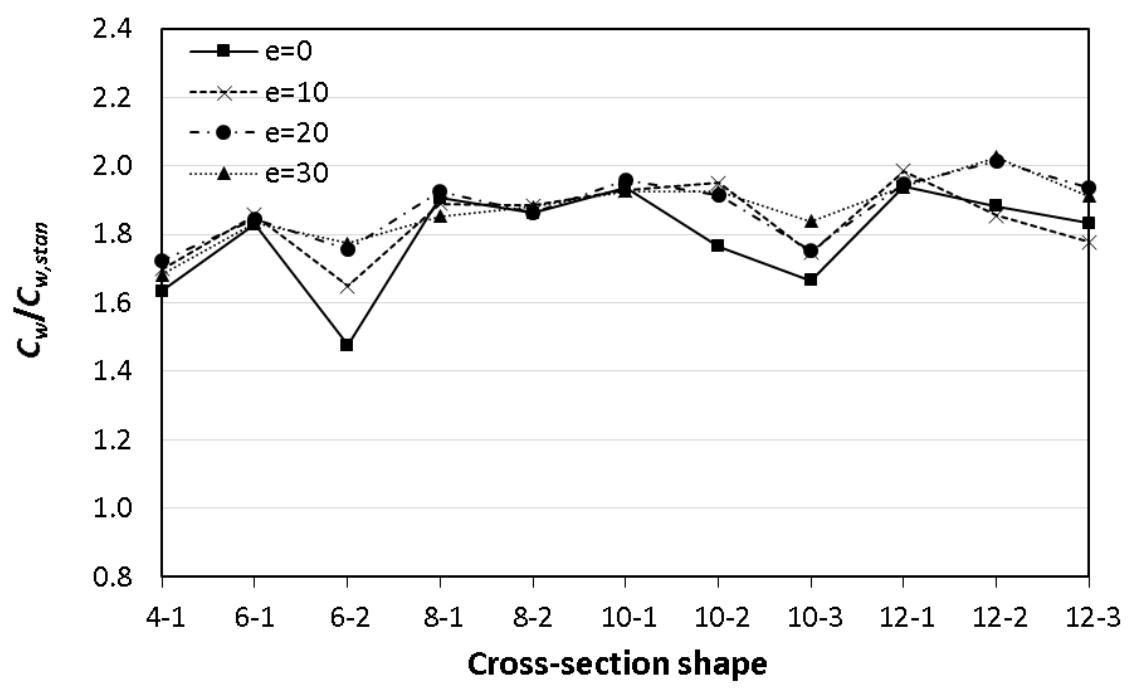

419

420

421

422

423

424

425

426

427

428

429

430

431

432

433

434

435

$\begin{cases}\varepsilon=\frac{\sigma}{E}+0.002\left(\frac{\sigma}{\sigma_{0.2}}\right)^{n s} & \text { for } \sigma \leq \sigma_{0.2} \\ \varepsilon=\varepsilon_{0.2}+\frac{100\left(\sigma-\sigma_{0.2}\right)}{E} & \text { for } \sigma \geq \sigma_{0.2}\end{cases}$

\section{Finite Element modelling}

\subsection{Material modelling and imperfections} the shape parameter $(n s)$ :

Fig. 15. Comparison between the warping constants of standard $Z$ and optimized cross-sections for the beam-columns with $3000 \mathrm{~mm}$ length

The purpose of this section is to validate the accuracy of the adopted optimization process based on DSM predictions and develop efficient analytical tools for the design and assessment of CFS beam-column elements. To this end, detailed nonlinear Finite Element (FE) models of the standard and optimized beamcolumn elements are developed using ABAQUS 6.14 software [41] by taking into account material nonlinearities and geometrical imperfections. It should be noted that the modelling techniques utilized in this paper, including the material behaviour, boundary conditions, the contact interaction, the analysis method and the meshing, are based on the models adopted by Yu and Schafer [48] and Ye et al. [31, 33], which have been extensively validated against experimental results for both column and beam elements.

To model the material properties of the beam-column members, the bi-linear stress-strain behaviour of steel plate proposed by Haidarali and Nethercot [49] was used, as shown in Fig. 16. This model is expressed in terms of elastic modulus $(E)$, the $0.2 \%$ proof stress $\left(\sigma_{0.2}\right)$, the total strain at $\sigma_{0.2}\left(\varepsilon_{0.2}\right)$, and

In this study, the elastic modulus and the $0.2 \%$ proof stress of the CFS material were taken as $E=210$ $437 \mathrm{GPa}$, and $\sigma_{0.2}=350 \mathrm{MPa}$, respectively. In addition, $n s$ was selected equal to 28 as recommended by Gardner and Ashraf [50] for grades 350 and 450 steel. 
441 The geometrical imperfections were predicted using elastic eigenvalue buckling analysis, available in

Fig. 16. Bi-linear stress-strain behaviour of CFS material used in FE modelling ABAQUS library [41]. The general shape of the dominant buckling mode, which can be either local, distortional, or global, was incorporated into the CFS elements and then scaled to the particular amplitude. The amplitude of imperfection for global buckling, which is in the shape of a half-sine wave, is taken as $L / 1000$ (where $L$ is the beam-column length) [27]. Based on the work conducted by Schafer and Peköz [51], the local and distortional buckling amplitudes were selected equal to $0.34 \mathrm{t}$ and $0.94 \mathrm{t}$, respectively, corresponding to a Cumulative Distribution Function (CDF) value of 50\%.

It should be noted that the effects of residual stresses and strain hardening of the round corners were not taken into account in the numerical models. While considering strain hardening of the round corners and the effects of the residual stresses may lead to slight changes in the ultimate capacity of the CFS elements $[31,33]$, it has been previously shown that they generally have opposite effects, and therefore, their influences should be either modelled or cancelled out together in thin-walled open sections [51, 52].

\subsection{Element type, meshing, loading and boundary conditions}

The CFS beam-column members were modelled in ABAQUS [41] using the S4R element, which is a 4noded quadrilateral shell element with reduced integration, while an 8-noded linear brick solid element with reduced integration and hourglass control (C3D8R) was utilised for modelling of end-plates. Following comprehensive mesh sensitivity analyses, a size of $10 \times 10 \mathrm{~mm}$ was chosen for the mesh as a further mesh refinement could not noticeably change the results.

Fig. 17 shows the developed FE model of the beam-column element with the imposed loading and boundary conditions. The supports at the two ends of the specimen were simulated using $30 \mathrm{~mm}$ thick end-plates by coupling the nodes at each end-plate to the reference point defined on the end-plate surface. While the centroid of the anti-symmetric cross-section was located on the centroid of the endplate, the value of eccentricity was allocated using the offset of the reference point from the centroid of

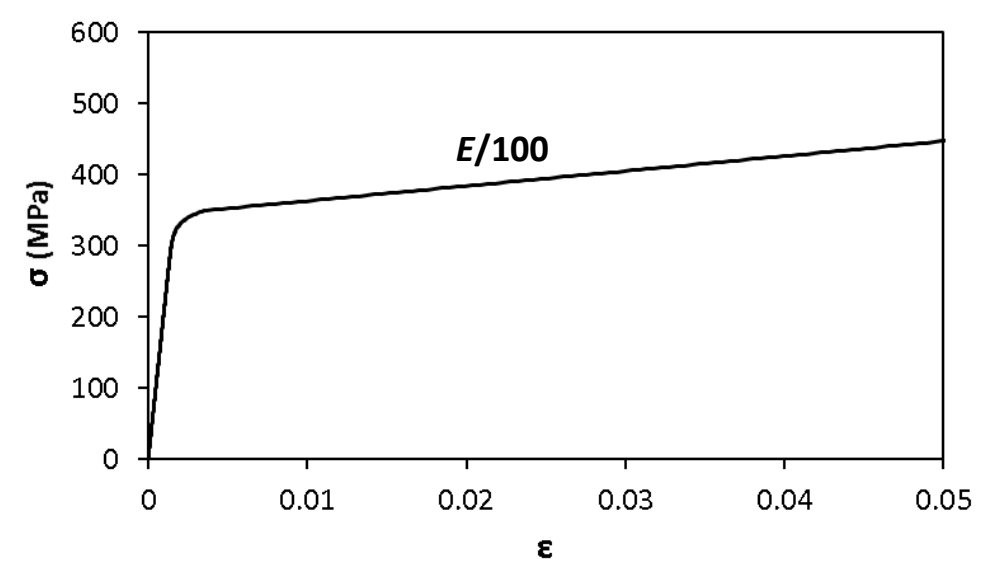


the end-plate in Y-direction (see Fig. 17). The axial compression load is applied to one end of the specimen (i.e. reference point) in a displacement control manner. A node-to-surface contact between the specimen and the end-plates was used to define the interaction of end sections and end-plates. The combined contact behaviour of "hard" and "rough" available in ABAQUS library was employed in the normal and tangential directions to avoid penetration of the surfaces into each other and restrain any tangential slip between the specimen and the end-plate. The presence of friction prevented the end sections from lateral expansions caused by Poisson effects. In this study, nonlinear inelastic post-buckling analysis was performed by using the standard RIKS arc-length method, available in Abaqus library [41], to estimate the capacity of the selected beam-column elements.

It should be noted that warping was restrained in the developed FE models since it is challenging to obtain the capacity of the beam-column elements with the free warping condition through FE simulation. However, the effect of warping was neglected in Section 3, where the capacity results have been predicted using DSM equations. This is due to the fact that the signature curve obtained from CUFSM software [42] is mainly established for the warping free elements. Previous experimental and analytical studies $[53,54]$ revealed that restrained warping boundary conditions lead to an increase in critical elastic distortional buckling of the CFS columns $\left(P_{c r d}\right)$ due to the shortening of the half-wave length, while the critical elastic local buckling $\left(P_{c r l}\right)$ remains unchanged. This strength enhancement can be taken into account through a boosting factor recommended by Moen [54]. In another study, Rajkannu and Jayachandran [55] modified the strength equation in DSM for global buckling of the axial compressive element $\left(P_{n e}\right)$ to consider the effect of restrained warping.

In this study, to address the above issue, the results of FE are compared with the results of DSM equations in which the elastic critical buckling load and bending moment were obtained from Nonlinear Elastic Buckling analysis on the restrained warping FE models. This leads to a more reasonable comparison between the results of FE and DSM as the restrained warping condition is reflected in both predictions. The FE model shown in Fig. 17 was adopted for this purpose, except that equal and opposite compressive load and bending moment were applied to both ends of the end-plates to achieve elastic critical buckling load $\left(P_{c r}^{F E}\right)$ and bending moment $\left(M_{c r}^{F E}\right)$, respectively. Depending on the dominant buckling mode shape obtained from FE elastic buckling analysis, either the local or the distortional DSM equation was adopted to calculate the maximum capacity of the elements. 


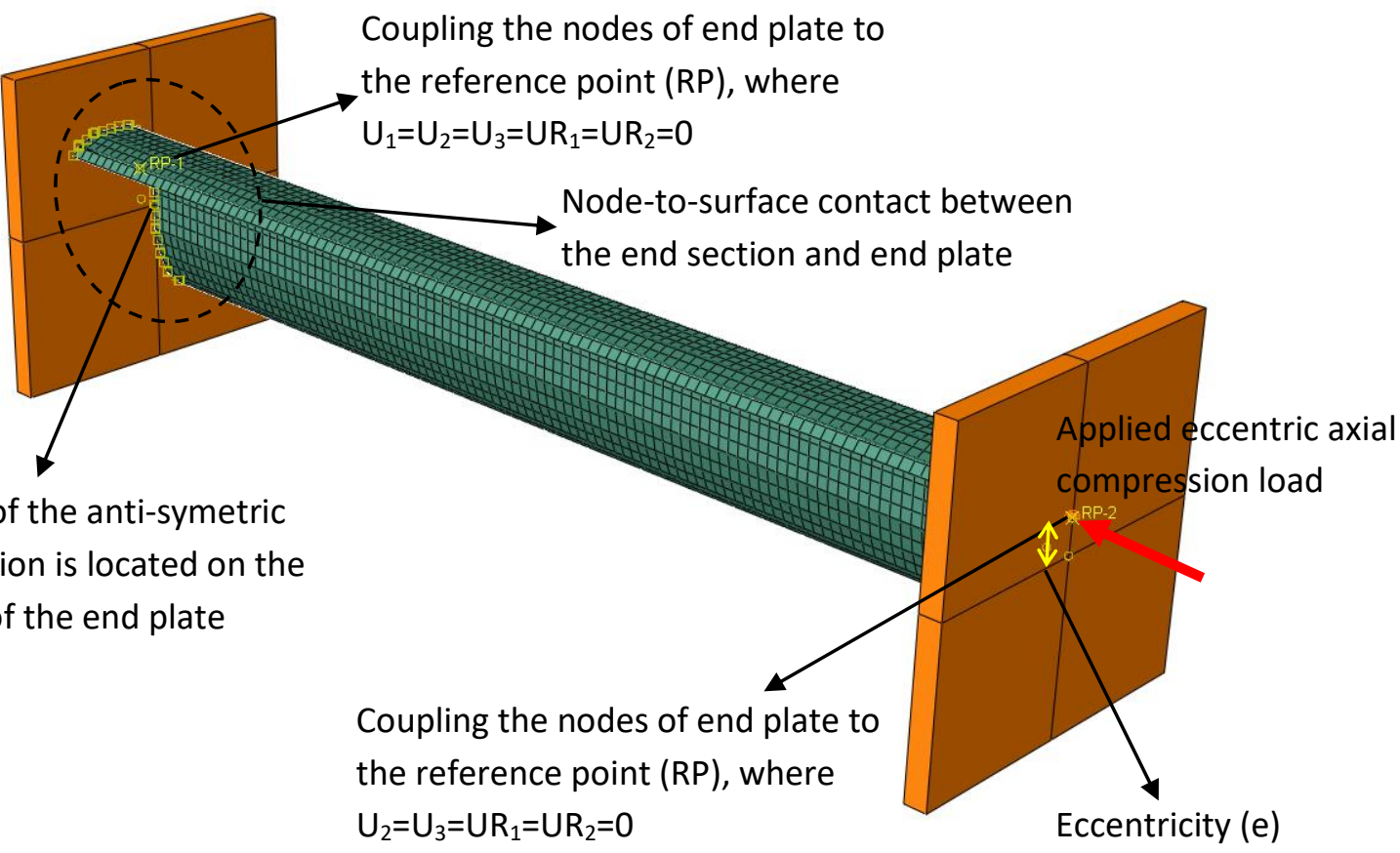

Fig. 17. Applied loading and boundary conditions of the beam-column member

Centriod of the anti-symetric cross-section is located on the centroid of the end plate

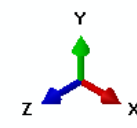

Coupling the nodes of end plate to the reference point (RP), where $\mathrm{U}_{2}=\mathrm{U}_{3}=\mathrm{UR}_{1}=\mathrm{UR}_{2}=0$
Eccentricity (e)

\subsection{Comparative FE and DSM results}

\subsubsection{FE vs DSM}

Tables 3 and 4 list the FE elastic critical buckling load $\left(P_{c r}^{F E}\right)$ and bending moment $\left(M_{c r}^{F E}\right)$, and maximum capacity obtained from DSM $\left(P^{D S M}\right)$ and FE $\left(P^{F E}\right)$ for eleven selected optimized cross-sections as well as the standard $Z$ section with the short and long length ( $L=1000 \mathrm{~mm}$ and $3000 \mathrm{~mm}$ ) and two different eccentricities ( $\mathrm{e}=0$ and $30 \mathrm{~mm}$ ). In general, the results obtained from DSM are shown to be acceptable for both short and long beam-column members with the average error less than $14 \%$. It can be also noted that the average error for the aforementioned cases shows slightly higher values for the beam-columns having higher load eccentricity. The ranges of strength ratios obtained from FE results to DSM predictions $\left(P^{F E} / P^{D S M}\right)$ vary from 0.88 to 1.34 , and from 0.9 to 1.41 for the short beam-column with $e=0 \mathrm{~mm}$ and $e=30 \mathrm{~mm}$, respectively. These ranges change from 0.8 to 1.17 , and from 0.76 to 1.10 for the long beamcolumns with the same axial load eccentricities. This highlights the need for the development of new approaches to improve the DSM reliability for the irregular shapes, especially for the beam-column elements under high load eccentricities.

\subsubsection{Comparative FE results for optimized sections}

The maximum capacities of the optimized beam-column elements to those with the standard $\mathrm{Z}$ section $\left(P^{F E} / P_{\text {stan }}^{F E}\right)$ are presented in Tables 3 and 4 based on the results of the detailed FE models. In general, the results follow a relatively similar trend as those obtained based on DSM discussed in Section 3. The 
results show that, for the given plate width and thickness, the adopted optimization process could increase the strength of beam-column members $(P)$ on average $43 \%$ and $34 \%$ for short beam-column members with $e=0 \mathrm{~mm}$ and $e=30 \mathrm{~mm}$, respectively. The efficiency of the optimization method increased to $12 \%$ and $19 \%$ for the long beam-column members with the same eccentricity levels.

Fig. 18 compares the compressive load versus shortening curves of the $1000 \mathrm{~mm}$ beam-column member with the standard and optimized 10-1 cross-sections for the eccentricity values of $e=0 \mathrm{~mm}$ and $e=30$ $\mathrm{mm}$. The results show that the proposed optimization algorithm could significantly increase both the ultimate capacity and stiffness of the beam-column elements. Fig. 19 also demonstrates the typical failure mode of the aforementioned CFS beam-column members, which is caused by either local, distortional or local-global buckling mode. The standard beam-columns under $e=0$ and $e=30 \mathrm{~mm}$ eccentric loads failed in local followed by distortional buckling modes, which are consistent with the failure modes predicted by CUFSM [42] (see Table A.1). However, distrortional buckling mode was observed to be dominant for the case of the corresponding optimized beam-column elements. In general, the results of this study indicate the adequacy and reliability of the proposed optimization framework as a practical tool for more efficient design of CFS beam-column elements.

Table 3. Comparison between the strength of $1000 \mathrm{~mm}$ CFS beam-column optimized and standard members with different eccentricities by considering fixed-warping boundary conditions.

\begin{tabular}{|c|c|c|c|c|c|c|c|c|c|c|c|c|}
\hline \multirow{2}{*}{$\begin{array}{l}\text { Section } \\
\text { shape }\end{array}$} & \multicolumn{6}{|c|}{$e=0 \mathrm{~mm}$} & \multicolumn{6}{|c|}{$e=30 \mathrm{~mm}$} \\
\hline & $P_{c r}^{F E}$ & $M_{c r}^{F E}$ & $P^{D S M}$ & $P^{F E}$ & $P^{F E} / P^{D S M}$ & $P^{F E} / P_{\text {stan }}^{F E}$ & $P_{c r}^{F E}$ & $M_{c r}^{F E}$ & $P^{D S M}$ & $P^{F E}$ & $P^{F E} / P^{D S M}$ & $P^{F E} / P_{\text {stan }}^{F E}$ \\
\hline $\begin{array}{l}\text { Standard } \\
\text { Z section }\end{array}$ & 8.9 & 2.8 & 38.4 & 50.1 & 1.30 & 1.00 & 8.9 & 2.8 & 30.0 & 40.1 & 1.34 & 1.00 \\
\hline 4-1 & 37.1 & 1.9 & 65.0 & 87.2 & 1.34 & 1.74 & 30.8 & 2.2 & 36.1 & 50.7 & 1.41 & 1.26 \\
\hline $6-1$ & 78.8 & 3.5 & 84.6 & 76.0 & 0.90 & 1.52 & 90.4 & 5.6 & 56.5 & 50.8 & 0.90 & 1.27 \\
\hline $6-2$ & 32.2 & 1.5 & 46.8 & 52.3 & 1.12 & 1.04 & 44.7 & 4.0 & 39.2 & 41.6 & 1.06 & 1.04 \\
\hline $8-1$ & 116.8 & 5.7 & 96.5 & 85.1 & 0.88 & 1.70 & 111.0 & 9.1 & 58.3 & 66.8 & 1.15 & 1.67 \\
\hline $8-2$ & 110.0 & 6.2 & 94.6 & 91.4 & 0.97 & 1.83 & 67.8 & 4.8 & 43.7 & 47.9 & 1.10 & 1.19 \\
\hline $10-1$ & 94.7 & 6.4 & 78.4 & 84.0 & 1.07 & 1.68 & 87.5 & 7.4 & 51.7 & 58.5 & 1.13 & 1.46 \\
\hline $10-2$ & 66.8 & 4.6 & 67.1 & 69.3 & 1.03 & 1.38 & 101.4 & 7.0 & 54.6 & 55.7 & 1.02 & 1.39 \\
\hline $10-3$ & 38.2 & 2.4 & 51.0 & 54.1 & 1.06 & 1.08 & 94.1 & 6.3 & 48.9 & 52.5 & 1.07 & 1.31 \\
\hline $12-1$ & 61.8 & 4.0 & 64.7 & 60.8 & 0.94 & 1.21 & 74.1 & 7.2 & 49.4 & 55.1 & 1.12 & 1.37 \\
\hline $12-2$ & 81.4 & 6.1 & 73.4 & 65.1 & 0.89 & 1.30 & 66.0 & 5.2 & 45.6 & 52.8 & 1.16 & 1.31 \\
\hline $12-3$ & 43.2 & 3.3 & 54.3 & 62.2 & 1.14 & 1.24 & 107.6 & 9.1 & 55.0 & 60.0 & 1.09 & 1.49 \\
\hline
\end{tabular}


Table 4. Comparison between the strength of $3000 \mathrm{~mm}$ CFS beam-column optimized and standard members with different eccentricities by considering fixed-warping boundary conditions.

\begin{tabular}{|c|c|c|c|c|c|c|c|c|c|c|c|c|}
\hline \multirow{2}{*}{$\begin{array}{l}\text { Section } \\
\text { shape }\end{array}$} & \multicolumn{6}{|c|}{$e=0 \mathrm{~mm}$} & \multicolumn{6}{|c|}{$e=30 \mathrm{~mm}$} \\
\hline & $P_{c r}^{F E}$ & $M_{c r}^{F E}$ & $P^{D S M}$ & $P^{F E}$ & $P^{F E} / P^{D S M}$ & $P^{F E} / P_{\text {stan }}^{F E}$ & $P_{c r}^{F E}$ & $M_{c r}^{F E}$ & $P^{D S M}$ & $P^{F E}$ & $P^{F E} / P^{D S M}$ & $P^{F E} / P_{\text {stan }}^{F E}$ \\
\hline $\begin{array}{l}\text { Standard } \\
\text { Z section }\end{array}$ & 8.9 & 2.8 & 38.5 & 40.2 & 1.04 & 1.00 & 8.9 & 2.8 & 30.0 & 28.9 & 0.96 & 1.00 \\
\hline 4-1 & 30.3 & 1.8 & 60.5 & 57.5 & 0.95 & 1.43 & 32.9 & 2.5 & 36.0 & 32.5 & 0.90 & 1.12 \\
\hline $6-1$ & 38.6 & 2.3 & 66.0 & 55.0 & 0.83 & 1.37 & 41.5 & 2.7 & 36.2 & 30.2 & 0.83 & 1.04 \\
\hline $6-2$ & 23.7 & 1.6 & 55.3 & 57.9 & 1.05 & 1.44 & 30.8 & 2.8 & 36.6 & 29.6 & 0.81 & 1.02 \\
\hline $8-1$ & 39.1 & 2.3 & 66.3 & 57.1 & 0.86 & 1.42 & 34.5 & 3.2 & 40.1 & 36.1 & 0.90 & 1.25 \\
\hline $8-2$ & 34.2 & 2.7 & 48.2 & 42.0 & 0.87 & 1.05 & 36.1 & 2.9 & 31.8 & 33.9 & 1.06 & 1.17 \\
\hline $10-1$ & 38.5 & 2.3 & 65.9 & 61.9 & 0.94 & 1.54 & 37.9 & 3.3 & 42.9 & 36.7 & 0.86 & 1.27 \\
\hline $10-2$ & 29.2 & 2.2 & 44.4 & 48.0 & 1.08 & 1.19 & 36.3 & 3.3 & 39.1 & 29.9 & 0.76 & 1.03 \\
\hline $10-3$ & 24.2 & 1.9 & 40.2 & 43.3 & 1.08 & 1.08 & 35.0 & 2.9 & 37.3 & 32.7 & 0.88 & 1.13 \\
\hline $12-1$ & 47.7 & 2.9 & 57.1 & 50.6 & 0.89 & 1.26 & 42.3 & 3.0 & 35.9 & 39.2 & 1.09 & 1.36 \\
\hline $12-2$ & 55.3 & 2.3 & 61.3 & 49.1 & 0.80 & 1.22 & 53.0 & 4.2 & 38.4 & 33.7 & 0.88 & 1.17 \\
\hline $12-3$ & 22.1 & 1.6 & 38.3 & 44.9 & 1.17 & 1.12 & 34.3 & 2.9 & 31.2 & 34.5 & 1.10 & 1.19 \\
\hline
\end{tabular}

534
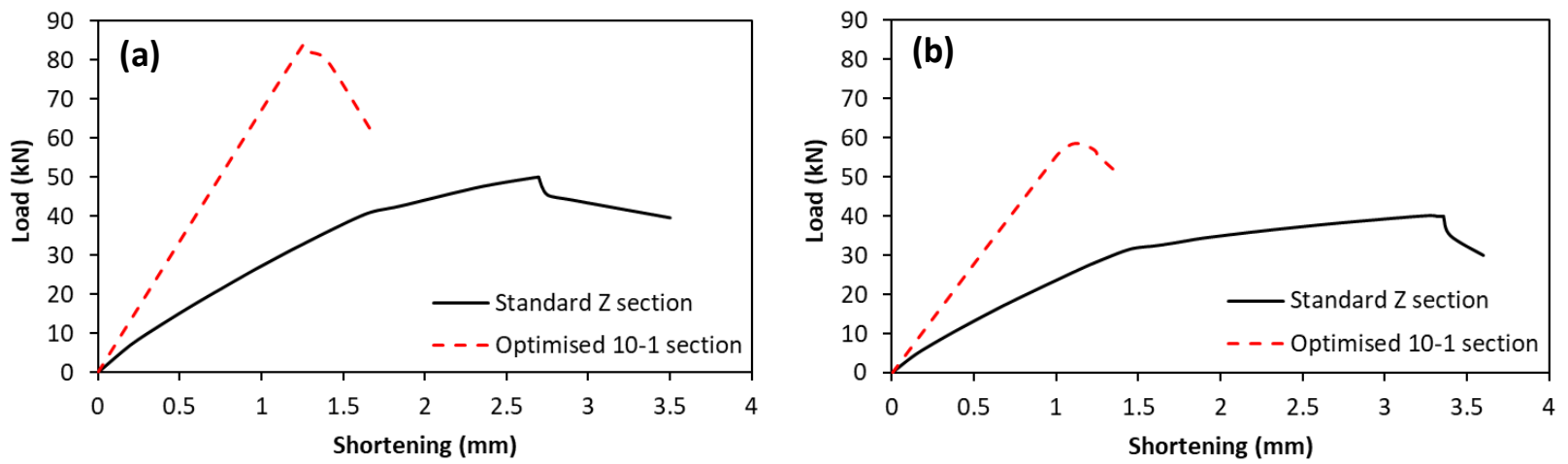

537 Fig. 18. Comparison between axial compression load versus shortening curves of the $1000 \mathrm{~mm}$ CFS beamcolumn members with standard and optimized 10-1 sections for two different load combinations: (a) e=0 
540

541

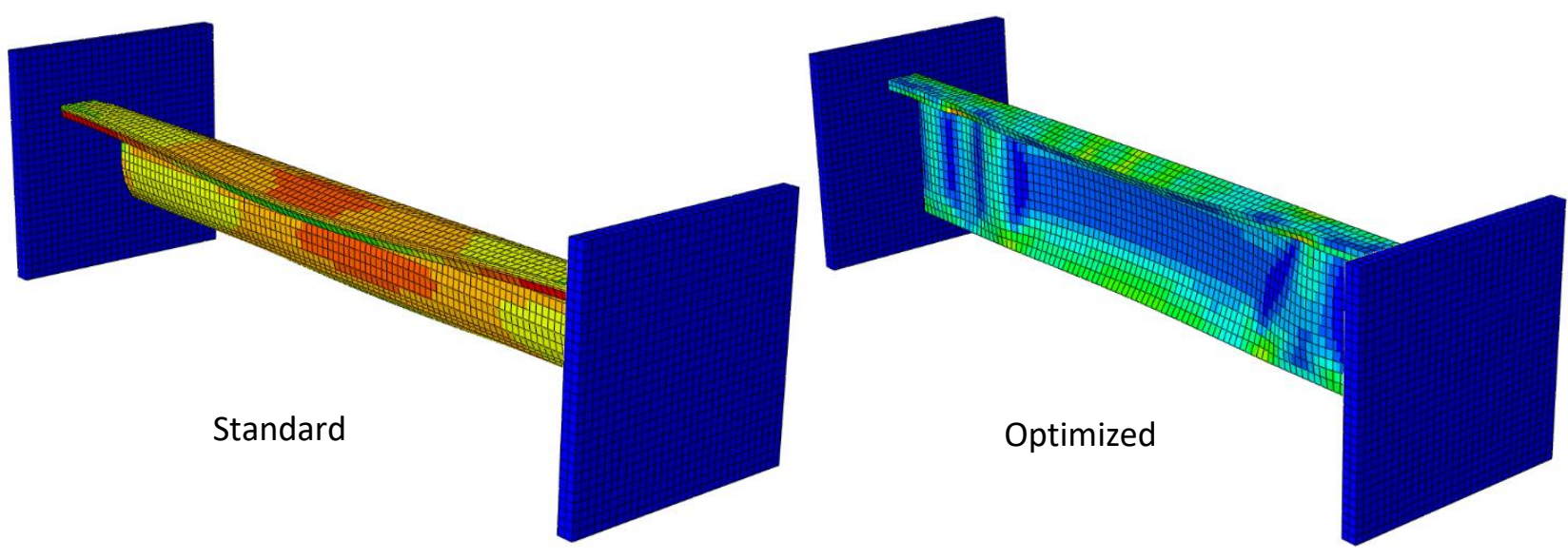

(a)

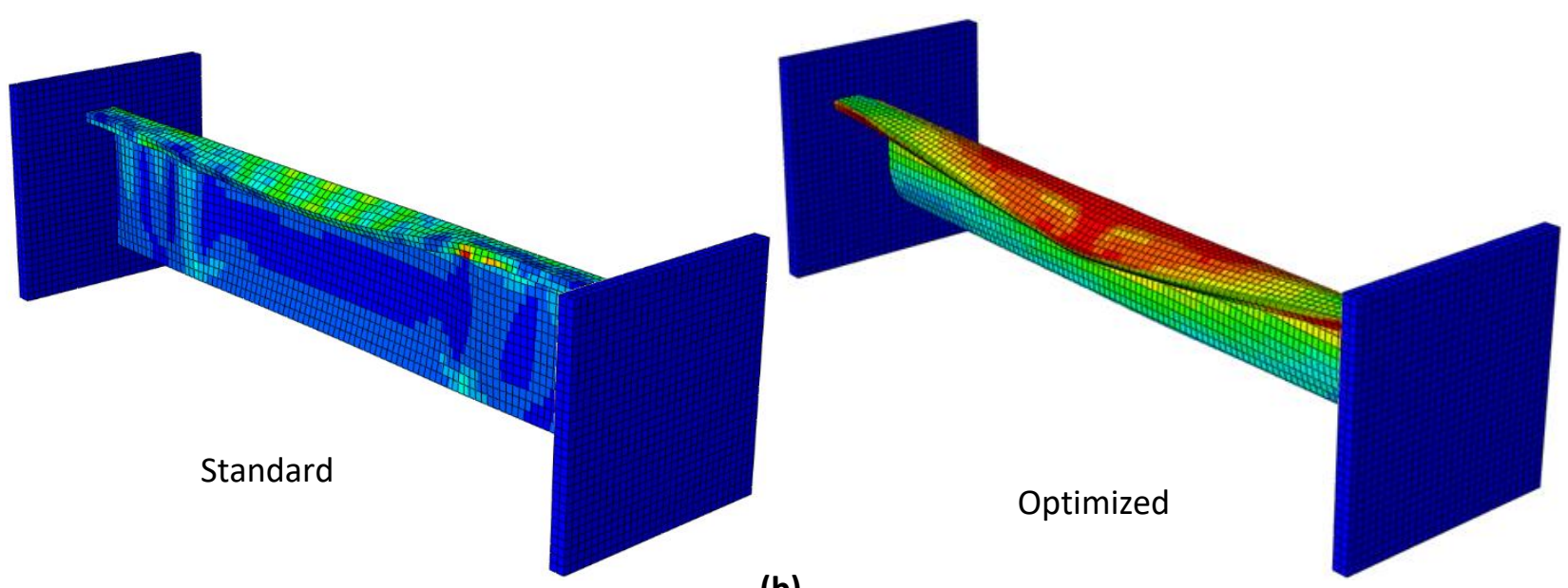

(b)

Fig. 19. Failure modes of $1000 \mathrm{~mm}$ CFS beam-column members with standard and optimized 10-1 sections for two different load combinations: (a) e=0 $\mathrm{mm}$ and (b) $30 \mathrm{~mm}$

\section{Summary and conclusion}

This paper presented a practical optimization methodology for pin-ended anti-symmetric CFS beamcolumns members subjected to various combinations of axial compression and bending moment. The optimization process was carried out using the GA method with respect to the buckling resistance of CFS beam-column elements determined according to the Direct Strength Method (DSM). Eleven crosssections with different number of fold-lines $(4,6,8,10,12)$ and three different element length (1000, $2000,3000 \mathrm{~mm}$ ) were considered. The length and angle of the constituent elements of the cross-sections (i.e. strips) were considered as main design variables, while a range of practical manufacturing and construction limitations were imposed. Detailed nonlinear Finite Element (FE) models were also developed using ABAQUS software to validate the accuracy of the adopted optimization process. Based on the results of this study, the following conclusions can be drawn:

- For the given number of foldings and lip strips, the optimum cross-sectional shapes were affected by the element length and value of axial load eccentricity. The optimum cross-sections tend to have a 
more spread shape when the eccentricity increases, especially in the short length elements. It was also shown that for the medium and long length members the general shape of the optimum solutions was less affected by increasing the eccentricity due to the dominant behaviour of the global buckling modes.

- $\quad$ For the given plate width and thickness, the adopted optimization process could significantly increase the strength of beam-column members with short, medium and long length on average by $62 \%, 92 \%$, and $188 \%$ compared to those with standard $Z$ section, respectively. It was shown that increasing the length of the beam-column members generally increased the efficiency of the optimization. The results also demonstrated that the warping constants of the majority of optimized sections were noticeably increased (up to $108 \%$ ).

- By increasing the number of lip strips, the optimized results for long beam-columns could be generally improved, while this was not necessarily the case for the short and medium length elements. This implied that increasing the number of lips in the optimum sections increased the global buckling resistance, which governed the results in the long length elements.

- The optimum cross-sectional shapes of the short members varied more significantly compared to the other lengths by changing the imposed practical constraints (i.e. number of rollers and lips' strips) and applied eccentricity, which can be attributed to the existence of different buckling modes for these sections. Besides, it was shown that the height of the beam-column cross-sections generally increased by increasing the level of eccentricity.

- The results of detailed nonlinear FE models showed that the warping restrained DSM predictions were acceptable for the short, medium, and long beam-column members with the average error less than $14 \%$. The capacities of the optimized beam-column elements to those with the standard $Z$ section, in general, followed a relatively similar trend as those obtained based on DSM. This demonstrates the reliability of the developed FE models as efficient analytical tools for the design and assessment of CFS beam-column elements.

\section{References}

[1] S. Torabian, B. Zheng, B.W. Schafer, Experimental response of cold-formed steel lipped channel beamcolumns, Thin-Walled Structures, 89 (2015) 152-168.

[2] S. Torabian, D.C. Fratamico, B.W. Schafer, Experimental response of cold-formed steel Zee-section beam-columns, Thin-Walled Structures, 98 (2016) 496-517.

[3] B. Zheng, X. Hua, G. Shu, Tests of cold-formed and welded stainless steel beam-columns, Journal of Constructional Steel Research, 111 (2015) 1-10.

[4] Y. Huang, B. Young, Experimental investigation of cold-formed lean duplex stainless steel beamcolumns, Thin-Walled Structures, 76 (2014) 105-117.

594 [5] S.M. Mojtabaei, M.Z. Kabir, I. Hajirasouliha, M. Kargar, Analytical and experimental study on the 595 seismic performance of cold-formed steel frames, Journal of Constructional Steel Research, 143 (2018) 596 18-31. 
[6] AISI S100-12, North American specification for the design of cold-formed steel structural members, American Iron and Steel Institute (AISI), Washington, DC, USA, (2012).

[7] W.-M. Lui, M. Ashraf, B. Young, Tests of cold-formed duplex stainless steel SHS beam-columns, Engineering Structures, 74 (2014) 111-121.

[8] CEN, Eurocode 3: Design of Steel Structures. Part 1-4: general rules: supplementary rules for stainless steels, in, Brussels: European Comittee for Standardization, (2006).

[9] SEI/ASCE-8-02, Specifications for the design of cold-formed stainless steel structural members American Society of Civil Engineers (ASCE), (2002).

[10] AS/NZS-4673, Cold-formed stainless steel structures. Australian Standard/New Zealand Standard., (2001).

[11] H. Liu, T. Igusa, B.W. Schafer, Knowledge-based global optimization of cold-formed steel columns, Thin-Walled Structures, 42 (2004) 785-801.

[12] J. Leng, J.K. Guest, B.W. Schafer, Shape optimization of cold-formed steel columns, Thin-Walled Structures, 49 (2011) 1492-1503.

[13] B.P. Gilbert, T.J.M. Savoyat, L.H. Teh, Self-shape optimisation application: Optimisation of coldformed steel columns, Thin-Walled Structures, 60 (2012) 173-184.

[14] B.P. Gilbert, L.H. Teh, H. Guan, Self-shape optimisation principles: Optimisation of section capacity for thin-walled profiles, Thin-Walled Structures, 60 (2012) 194-204.

[15] P. Sharafi, L.H. Teh, M.N.S. Hadi, Shape optimization of thin-walled steel sections using graph theory and ACO algorithm, Journal of Constructional Steel Research, 101 (2014) 331-341.

[16] J.F.A. Madeira, J. Dias, N. Silvestre, Multiobjective optimization of cold-formed steel columns, ThinWalled Structures, 96 (2015) 29-38.

[17] B. Wang, G.L. Bosco, B.P. Gilbert, H. Guan, L.H. Teh, Unconstrained shape optimisation of singlysymmetric and open cold-formed steel beams and beam-columns, Thin-Walled Structures, 104 (2016) 5461.

[18] H. Adeli, A. Karim, Neural network model for optimization of cold-formed steel beams, J. Struct. Eng. ASCE 123, (1997) 1535-1543.

[19] A. Karim, H. Adeli, Global optimum design of cold-formed steel hat-shape beams, Thin-Walled Structures, 35 (1999) 275-288.

[20] Y.S. Tian, T.J. Lu, Minimum weight of cold-formed steel sections under compression, Thin-Walled Structures, 42 (2004) 515-532.

[21] J. Lee, S.M. Kim, H.S. Park, B.H. Woo, Optimum design of cold-formed steel channel beams using micro-genetic algorithm, Eng. Struct, 27 (2005) 17-24.

[22] K. Magnucki, M. Rodak, J. Lewiński, Optimization of mono- and anti-symmetrical I-sections of coldformed thin-walled beams, Thin-Walled Structures, 44 (2006) 832-836.

[23] T. Tran, L.-y. Li, Global optimization of cold-formed steel channel sections, Thin-Walled Structures, 44 (2006) 399-406.

[24] J. Lee, S.M. Kim, H.S. Park, B.H. Woo, Optimum design of cold-formed steel columns by using micro genetic algorithms, Thin-Walled Structures, 44 (2006) 952-960.

[25] M.M. Pastor, M. Casafont, E. Chillarón, A. Lusa, F. Roure, M.R. Somalo, Optimization of cold-formed steel pallet racking cross-sections for flexural-torsional buckling with constraints on the geometry, Engineering Structures, 31 (2009) 2711-2722.

[26] J. Leng, Z. Li, J.K. Guest, B.W. Schafer, Shape optimization of cold-formed steel columns with fabrication and geometric end-use constraints, Thin-Walled Structures, 85 (2014) 271-290.

[27] W. Ma, J. Becque, I. Hajirasouliha, J. Ye, Cross-sectional optimization of cold-formed steel channels to Eurocode 3, Engineering Structures, 101 (2015) 641-651.

[28] J. Ye, I. Hajirasouliha, J. Becque, A. Eslami, Optimum design of cold-formed steel beams using Particle Swarm Optimisation method, Journal of Constructional Steel Research, 122 (2016) 80-93.

[29] B. Wang, B.P. Gilbert, H. Guan, L.H. Teh, Shape optimisation of manufacturable and usable coldformed steel singly-symmetric and open columns, Thin-Walled Structures, 109 (2016) 271-284.

[30] B. Wang, B.P. Gilbert, A.M. Molinier, H. Guan, L.H. Teh, Shape optimisation of cold-formed steel columns with manufacturing constraints using the Hough transform, Thin-Walled Structures, 106 (2016) 75-92. 
[31] J. Ye, S.M. Mojtabaei, I. Hajirasouliha, Local-flexural interactive buckling of standard and optimised cold-formed steel columns, Journal of Constructional Steel Research, 144 (2018) 106-118.

[32] J. Ye, J. Becque, I. Hajirasouliha, S.M. Mojtabaei, J.B.P. Lim, Development of optimum cold-formed steel sections for maximum energy dissipation in uniaxial bending, Engineering Structures, 161 (2018) 5567.

[33] J. Ye, S.M. Mojtabaei, I. Hajirasouliha, P. Shepherd, K. Pilakoutas, Strength and deflection behaviour of cold-formed steel back-to-back channels, Engineering Structures, 177 (2018) 641-654.

[34] S.M. Mojtabaei, J. Ye, I. Hajirasouliha, Development of optimum cold-formed steel beams for serviceability and ultimate limit states using Big Bang-Big Crunch optimisation, Engineering Structures, 195 (2019) 172-181.

[35] J. Ye, I. Hajirasouliha, J. Becque, K. Pilakoutas, Development of more efficient cold-formed steel channel sections in bending, Thin-Walled Structures, 101 (2016) 1-13.

[36] D.T. Phan, S.M. Mojtabaei, I. Hajirasouliha, J. Ye, J.B.P. Lim, Coupled element and structural level optimisation framework for cold-formed steel frames, Journal of Constructional Steel Research, (2019) 105867.

[37] D.T. Phan, S.M. Mojtabaei, I. Hajirasouliha, T.L. Lau, J.B.P. Lim, Design and Optimization of ColdFormed Steel Sections in Bolted Moment Connections Considering Bimoment, Journal of Structural Engineering, 146 (2020) 04020153.

[38] J.M. Davies, Recent research advances in cold-formed steel structures, Journal of Constructional Steel Research, 55 (2000) 267-288.

[39] H. Parastesh, I. Hajirasouliha, H. Taji, A. Bagheri Sabbagh, Shape optimization of cold-formed steel beam-columns with practical and manufacturing constraints, Journal of Constructional Steel Research, 155 (2019) 249-259.

[40] AISI S100-16, North American specification for the design of cold-formed steel structural members. , American Iron and Steel Institute (AISI), Washington, DC, USA, (2016).

[41] Abaqus/CAE User's Manual, version 6.14-2, USA, (2014).

[42] B.W. Schafer, CUFSM Version 3.12, Department of Civil Engineering, Johns Hopkins University, http://www.ce.jhu.edu/bschafer/cufsm/, (2006).

[43] O. Yeniay, Penalty function methods for constrained optimization with genetic algorithms, Mathematical and Computational Applications, 10(1) (2005) 45-56.

[44] N.D. Lagaros, M. Papadrakakis, G. Kokossalakis, Structural optimization using evolutionary algorithms, Computers \& Structures, 80 (2002) 571-589.

[45] S. Ádány, B.W. Schafer, Buckling mode decomposition of single-branched open cross-section members via finite strip method: Application and examples, Thin-Walled Structures, 44 (2006) 585-600.

[46] S. Ádány, B.W. Schafer, Buckling mode decomposition of single-branched open cross-section members via finite strip method: Derivation, Thin-Walled Structures, 44 (2006) 563-584.

[47] Mathworks, Matlab R2011a, in, Mathworks, Inc, 2011.

[48] C. Yu, B.W. Schafer, Distortional Buckling Tests on Cold-Formed Steel Beams, Journal of Structural Engineering, 132 (2006) 515-528.

[49] M.R. Haidarali, D.A. Nethercot, Finite element modelling of cold-formed steel beams under local buckling or combined local/distortional buckling, Thin Wall Struct, 49 (2011) 1554-1562.

[50] L. Gardner, M. Ashraf, Structural design for non-linear metallic materials, Eng Struct, 28 (2006) 926934.

[51] B.W. Schafer, T. Peköz, Computational modeling of cold-formed steel: characterizing geometric imperfections and residual stresses, Journal of Constructional Steel Research, 47 (1998) 193-210.

[52] B.W. Schafer, Z. Li, C.D. Moen, Computational modeling of cold-formed steel, Thin-Walled Structures, 48 (2010) 752-762.

[53] B.W. Schafer, S. Ádány, Buckling analysis of cold-formed steel members using CUFSM: conventional and constrained finite strip methods, Eighteenth International Specialty Conference on Cold-Formed Steel Structures, Orland, (2006).

[54] C.D. Moen, Direct Strength Design for Cold-Formed Steel Members with Perforations, PhD thesis, John-Hopkins University (2008). 
702

703

704

705

706 707 Tables.

708 709

[55] J.S. Rajkannu, S.A. Jayachandran, Flexural-torsional buckling strength of thin-walled channel sections with warping restraint, Journal of Constructional Steel Research, 169 (2020) 106041.

\section{Appendix A}

The characteristics of the optimized cross-sections discussed in Section 3 were listed in the following

Table A1. Cross-sectional properties, nominal compressive and flexural capacities, and strength of beamcolumns with $1000 \mathrm{~mm}$ length

\begin{tabular}{|c|c|c|c|c|c|c|c|c|c|c|}
\hline $\begin{array}{c}\mathrm{e} \\
(\mathrm{mm})\end{array}$ & $\begin{array}{l}\text { Cross- } \\
\text { section } \\
\text { shape }\end{array}$ & $\begin{array}{c}h \\
(\mathrm{~mm})\end{array}$ & $\begin{array}{c}I_{x} \\
\left(\mathrm{~mm}^{4}\right)\end{array}$ & $\begin{array}{c}I_{y} \\
\left(\mathrm{~mm}^{4}\right)\end{array}$ & $\begin{array}{c}I_{1} \\
\left(\mathrm{~mm}^{4}\right)\end{array}$ & $\begin{array}{c}I_{2} \\
\left(\mathrm{~mm}^{4}\right)\end{array}$ & $\begin{array}{c}C_{w} \times 10^{6} \\
\left(\mathrm{~mm}^{6}\right)\end{array}$ & $\begin{array}{c}P_{n} \\
(\mathrm{kN})\end{array}$ & $\begin{array}{c}M_{n x} \\
\text { (kN.m) }\end{array}$ & $\begin{array}{c}P \\
(\mathrm{kN})\end{array}$ \\
\hline \multirow{12}{*}{0} & Z section & 200 & 1823917 & 121500 & 1884701 & 60716 & 933 & 39.7 (L) & 3.9 (L) & 39.7 \\
\hline & $4-1$ & 94 & 486000 & 631000 & 1012353 & 105129 & 880 & 65.0 (L) & $3.2(\mathrm{~L})$ & 65.0 \\
\hline & $6-1$ & 105.7 & 584000 & 1090000 & 1585946 & 90165 & 1160 & 83.8 (L) & 3.4 (L) & 83.8 \\
\hline & $6-2$ & 69.4 & 244000 & 1280000 & 1411227 & 116624 & 1012 & 48.1 (D) & 1.7 (D) & 42.0 \\
\hline & $8-1$ & 138.3 & 987000 & 824000 & 1697357 & 111331 & 1486 & $91.4(\mathrm{~L}-\mathrm{G})$ & 5.0 (D) & 91.4 \\
\hline & $8-2$ & 114.9 & 686000 & 929000 & 1469734 & 145823 & 1599 & 95.9 (L-G) & 4.2 (D) & 95.9 \\
\hline & $10-1$ & 149.2 & 1148000 & 727000 & 1756097 & 119073 & 1591 & $92.6(\mathrm{~L}-\mathrm{G})$ & 5.4 (D) & 79.4 \\
\hline & $10-2$ & 123.5 & 693000 & 794000 & 1312451 & 174468 & 1908 & 66.5 (D) & $3.3(\mathrm{~L})$ & 66.5 \\
\hline & $10-3$ & 107.9 & 524000 & 834000 & 1182688 & 175849 & 1410 & 46.1 (D) & 2.5 (D) & 46.1 \\
\hline & $12-1$ & 150.3 & 116000 & 810000 & 1857189 & 120062 & 1627 & 50.4 (D) & 4.6 (D) & 50.4 \\
\hline & $12-2$ & 114.4 & 592000 & 925000 & 1353023 & 164610 & 1898 & 72.3 (D) & 2.9 (D) & 72.3 \\
\hline & $12-3$ & 119 & 609000 & 759000 & 1185773 & 182253 & 1813 & 54.9 (D) & $2.6(\mathrm{~L})$ & 54.9 \\
\hline \multirow{14}{*}{10} & Z section & 200 & 1823917 & 121500 & 1884701 & 60716 & 933 & 39.7 (L) & 3.9 (L) & 33.3 \\
\hline & $4-1$ & 100.4 & 534000 & 504000 & 939594 & 99728 & 840 & 62.8 (L) & 3.5 (L) & 52.8 \\
\hline & $6-1$ & 144.9 & 1050000 & 635000 & 1585869 & 99592 & 1353 & 74.5 (D) & 4.7 (D) & 63.9 \\
\hline & $6-2$ & 72.2 & 292000 & 1300000 & 1480961 & 113471 & 1020 & 72.7 (D) & $2.2(D)$ & 53.1 \\
\hline & $8-1$ & 165 & 1320000 & 600000 & 1824058 & 107201 & 1563 & 83.0 (D) & 5.6 (D) & 72.0 \\
\hline & $8-2$ & 130.9 & 860000 & 743000 & 1442757 & 160863 & 1779 & 80.1 (D) & $4.3(D)$ & 67.1 \\
\hline & $10-1$ & 162.3 & 1300000 & 670000 & 1864562 & 110317 & 1568 & 55.4 (D) & 5.6 (D) & 50.4 \\
\hline & $10-2$ & 146.6 & 1030000 & 710000 & 1601432 & 140151 & 1831 & 95.3 (L-G) & 4.7 (D) & 74.9 \\
\hline & $10-3$ & 108 & 587000 & 990000 & 1413026 & 163217 & 1704 & 97.5 (L-G) & 3.5 (D) & 75.1 \\
\hline & $12-1$ & 161.2 & 1290000 & 668000 & 1850417 & 107736 & 1526 & 54.7 (D) & 5.4 (D) & 49.5 \\
\hline & $12-2$ & 150.3 & 1120000 & 610000 & 1590309 & 144376 & 1781 & 48.3 (D) & 4.6 (D) & 43.6 \\
\hline & $12-3$ & 145.6 & 1060000 & 674000 & 1594982 & 140514 & 1764 & 55.1 (D) & 5.1 (D) & 49.6 \\
\hline & Z sectio & 200 & 1823917 & 121500 & 1884701 & 60716 & 933 & 39.7 (L) & 3.9 (L) & 30.6 \\
\hline & 4-1 & 101 & 540000 & 560000 & 987704 & 113047 & 989 & $62.5(\mathrm{~L})$ & 3.4 (L) & 45.2 \\
\hline
\end{tabular}




\begin{tabular}{|c|c|c|c|c|c|c|c|c|c|c|}
\hline & $6-1$ & 138.4 & 972000 & 625000 & 1496998 & 100513 & 1299 & 85.1 (L) & 4.6 (D) & 61.7 \\
\hline & $6-2$ & 79.2 & 346000 & 1180000 & 1397711 & 131052 & 1163 & 50.7 (D) & 2.6 (D) & 35.9 \\
\hline & $8-1$ & 177.6 & 1490000 & 515000 & 1919629 & 91232 & 1431 & 87.4 (L-G) & 5.9 (D) & 67.0 \\
\hline \multirow[t]{13}{*}{20} & $8-2$ & 131.5 & 866000 & 713000 & 1413580 & 164706 & 1748 & 50.0 (D) & 4.2 (D) & 40.2 \\
\hline & $10-1$ & 176 & 1480000 & 570000 & 1950040 & 102654 & 1542 & 53.5 (D) & 5.6 (D) & 44.8 \\
\hline & $10-2$ & 160.1 & 1280000 & 540000 & 1696766 & 129196 & 1666 & 44.6 (D) & 4.8 (D) & 37.5 \\
\hline & $10-3$ & 123.1 & 700000 & 754000 & 1282747 & 172126 & 1642 & 49.9 (D) & 3.3 (D) & 38.0 \\
\hline & $12-1$ & 173.9 & 1450000 & 592000 & 1944390 & 103317 & 1552 & 53.9 (D) & 5.6 (D) & 45.1 \\
\hline & $12-2$ & 160.4 & 1270000 & 552000 & 1691001 & 128558 & 1705 & 48.7 (D) & 5.1 (D) & 40.7 \\
\hline & $12-3$ & 151.1 & 1130000 & 625000 & 1620532 & 133523 & 1752 & 94.5 (L-G) & 5.2 (D) & 68.9 \\
\hline & section & 200 & 1823917 & 121500 & 1884701 & 60716 & 933 & 39.7 (L) & 3.9 (L) & 28.4 \\
\hline & $4-1$ & 103.8 & 557000 & 496000 & 944715 & 108461 & 956 & 61.1 (L) & 3.5 (L) & 39.5 \\
\hline & $6-1$ & 159.7 & 1250000 & 516000 & 1676404 & 92357 & 1318 & 85.9 (L) & $5.1(\mathrm{~L})$ & 50.9 \\
\hline & $6-2$ & 106.7 & 434000 & 975000 & 1265816 & 144095 & 1251 & 68.7 (L) & $2.8(\mathrm{~L})$ & 39.0 \\
\hline & 8-1 & 180.3 & 1510000 & 545000 & 1969529 & 84892 & 1371 & 85.8 (L-G) & 5.9 (D) & 55.0 \\
\hline & $8-2$ & 137.6 & 935000 & 674000 & 1450471 & 159151 & 1784 & 50.5 (D) & 4.4 (D) & 37.4 \\
\hline \multirow[t]{6}{*}{30} & $10-1$ & 172 & 1420000 & 576000 & 1896313 & 103163 & 1529 & 56.2 (D) & 5.8 (D) & 43.4 \\
\hline & $10-2$ & 166.8 & 1310000 & 610000 & 1814299 & 110127 & 1653 & 85.3 (D) & $5.2(\mathrm{~L})$ & 56.8 \\
\hline & $10-3$ & 127.3 & 810000 & 800000 & 1447896 & 159921 & 1787 & 85.5 (D) & 4.3 (D) & 52.7 \\
\hline & $12-1$ & 180.3 & 1530000 & 511000 & 1944951 & 95380 & 1472 & 53.8 (D) & 6.0 (D) & 42.2 \\
\hline & $12-2$ & 166.5 & 1360000 & 577000 & 1812107 & 120975 & 1724 & 50.8 (D) & $5.6(\mathrm{~L})$ & 39.8 \\
\hline & $12-3$ & 156.8 & 1190000 & 633000 & 1700 & 1227 & 1719 & 93.2 (L-G) & 5.3 (D) & 57.4 \\
\hline
\end{tabular}

710

711 Table A2. Cross-sectional properties, nominal compressive and flexural capacities, and strength of beamcolumns with $2000 \mathrm{~mm}$ length

\begin{tabular}{|c|c|c|c|c|c|c|c|c|c|c|}
\hline $\begin{array}{c}\mathrm{e} \\
(\mathrm{mm})\end{array}$ & $\begin{array}{l}\text { Cross- } \\
\text { section } \\
\text { shape }\end{array}$ & $\begin{array}{c}h \\
(\mathrm{~mm})\end{array}$ & $\begin{array}{c}I_{x} \\
\left(\mathrm{~mm}^{4}\right)\end{array}$ & $\begin{array}{c}I_{y} \\
\left(\mathrm{~mm}^{4}\right)\end{array}$ & $\begin{array}{c}I_{1} \\
\left(\mathrm{~mm}^{4}\right)\end{array}$ & $\begin{array}{c}I_{2} \\
\left(\mathrm{~mm}^{4}\right)\end{array}$ & $\begin{array}{c}C_{w} \times 10^{6} \\
\left(\mathrm{~mm}^{6}\right)\end{array}$ & $\begin{array}{c}P_{n} \\
(\mathrm{kN})\end{array}$ & $\begin{array}{c}M_{n x} \\
\text { (kN.m) }\end{array}$ & $\underset{(\mathrm{kN})}{P}$ \\
\hline & Z section & 200 & 1823917 & 121500 & 1884701 & 60716 & 933 & $27.6(\mathrm{~L}-\mathrm{G})$ & 1.9 (L) & 27 \\
\hline & $4-1$ & 94.2 & 496000 & 788000 & 1152138 & 132513 & 1188 & $56.6(\mathrm{~L}-\mathrm{G})$ & $2.6(\mathrm{~L})$ & 56. \\
\hline & $6-1$ & 129.1 & 900000 & 792000 & 1544338 & 148629 & 1683 & 54.6 (D) & $3.4(\mathrm{~L})$ & \\
\hline & $6-2$ & 83.9 & 351000 & 1036000 & 1230476 & 157109 & 1324 & 45.4 (D) & 2.1 (D) & \\
\hline & 8-1 & 127.2 & 864000 & 751000 & 1457982 & 157609 & 1718 & 57.2 (D) & 3.4 (L) & 57 \\
\hline 0 & $8-2$ & 111.7 & 548000 & 775000 & 1131804 & 191695 & 1735 & 49.0 (D) & $2.3(\mathrm{~L})$ & \\
\hline & $10-1$ & 127.2 & 860000 & 793000 & 1500570 & 154680 & 1746 & 57.6 (D) & 3.3 (L) & \\
\hline & $10-2$ & 110.5 & 512000 & 750000 & 1062070 & 200212 & 1669 & 44.7 (D) & $2.2(D)$ & \\
\hline & $10-3$ & 115.2 & 539000 & 658000 & 985156 & 211554 & 1561 & 36.3 (D) & $2.1(\mathrm{~L})$ & 43 \\
\hline & $12-1$ & 132.9 & 924000 & 787000 & 1562951 & 149131 & 1755 & 56.6 (D) & $3.3(\mathrm{~L})$ & \\
\hline & $12-2$ & 115.1 & 595000 & 754000 & 1157421 & 191844 & 1865 & 59.5 (D) & $2.6(\mathrm{~L})$ & \\
\hline
\end{tabular}


12-3 $111.5 \quad 5430007950001142642195309 \quad 1761 \quad 42.0$ (D) 2.0 (L) 42.0

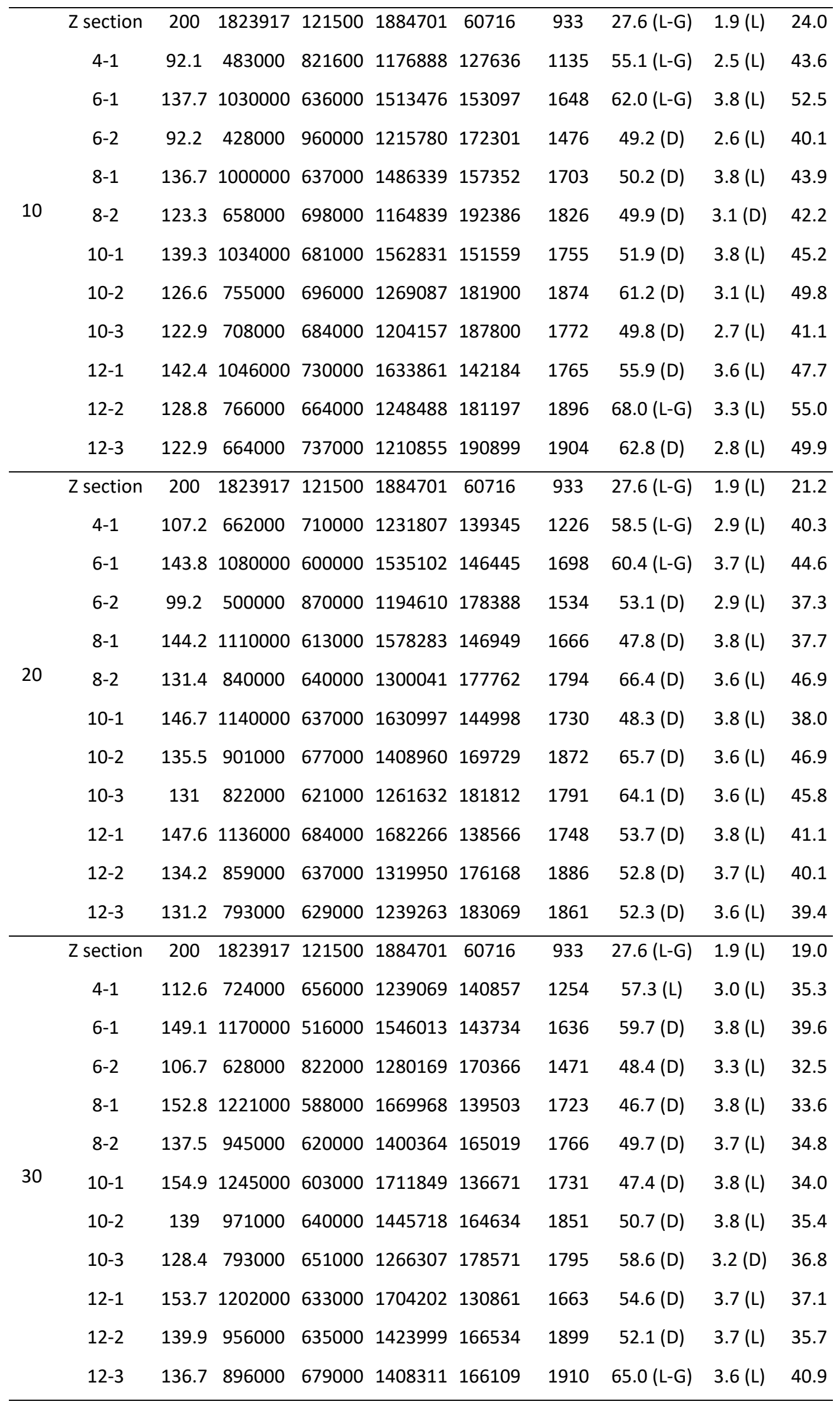


Table A3. Cross-sectional properties, nominal compressive and flexural capacities, and strength of beamcolumns with $3000 \mathrm{~mm}$ length

\begin{tabular}{|c|c|c|c|c|c|c|c|c|c|c|}
\hline $\begin{array}{c}\mathrm{e} \\
(\mathrm{mm})\end{array}$ & $\begin{array}{l}\text { Cross- } \\
\text { section } \\
\text { shape }\end{array}$ & $\begin{array}{c}h \\
(\mathrm{~mm})\end{array}$ & $\begin{array}{c}I_{x} \\
\left(\mathrm{~mm}^{4}\right)\end{array}$ & $\begin{array}{c}I_{y} \\
\left(\mathrm{~mm}^{4}\right)\end{array}$ & $\begin{array}{c}I_{1} \\
\left(\mathrm{~mm}^{4}\right)\end{array}$ & $\begin{array}{c}I_{2} \\
\left(\mathrm{~mm}^{4}\right)\end{array}$ & $\begin{array}{c}C_{w} \times 10^{6} \\
\left(\mathrm{~mm}^{6}\right)\end{array}$ & $\begin{array}{c}P_{n} \\
(\mathrm{kN})\end{array}$ & $\begin{array}{c}M_{n x} \\
\text { (kN.m) }\end{array}$ & $P(\mathrm{kN})$ \\
\hline \multirow{12}{*}{0} & Z section & 200 & 1823917 & 121500 & 1884701 & 60716 & 933 & $12.3(\mathrm{~L}-\mathrm{G})$ & $0.9(\mathrm{~L}-\mathrm{G})$ & 12.3 \\
\hline & $4-1$ & 97.7 & 503000 & 831000 & 1162524 & 170845 & 1525 & 34.5 (L-G) & $1.6(\mathrm{~L})$ & 34.5 \\
\hline & $6-1$ & 121.7 & 776000 & 685000 & 1286859 & 174229 & 1706 & $35.2(\mathrm{~L}-\mathrm{G})$ & 1.9 (L) & 35.2 \\
\hline & $6-2$ & 94.8 & 378000 & 844000 & 1027039 & 195112 & 1378 & 38.8 (D) & $1.8(\mathrm{~L}-\mathrm{G})$ & 38.8 \\
\hline & 8-1 & 126 & 820000 & 681000 & 1328694 & 172339 & 1778 & 34.8 (L-G) & 1.9 (L) & 34.8 \\
\hline & $8-2$ & 114 & 560000 & 706000 & 1059061 & 207717 & 1737 & $42.0(\mathrm{~L}-\mathrm{G})$ & 1.9 (L) & 42.0 \\
\hline & $10-1$ & 123.7 & 786000 & 754000 & 1371155 & 168842 & 1806 & 34.1 (L-G) & $1.9(\mathrm{~L})$ & 34.1 \\
\hline & $10-2$ & 110.6 & 515000 & 718000 & 1028910 & 204541 & 1647 & $41.3(\mathrm{~L}-\mathrm{G})$ & $1.9(\mathrm{~L})$ & 41.3 \\
\hline & $10-3$ & 112.1 & 514000 & 644000 & 942461 & 215352 & 1554 & 38.9 (D) & $2.1(\mathrm{~L}-\mathrm{G})$ & 38.9 \\
\hline & $12-1$ & 130.9 & 900000 & 775000 & 1517354 & 157996 & 1810 & 31.9 (L-G) & $2.0(\mathrm{~L}-\mathrm{G})$ & 31.9 \\
\hline & $12-2$ & 112.1 & 545000 & 758000 & 1108111 & 195580 & 1756 & 39.5 (L-G) & $1.7(\mathrm{~L})$ & 39.5 \\
\hline & $12-3$ & 118.2 & 566000 & 684000 & 1045311 & 204549 & 1709 & 40.1 (D) & $1.6(\mathrm{~L})$ & 40.1 \\
\hline \multirow{12}{*}{10} & section & 200 & 1823917 & 121500 & 1884701 & 60716 & 933 & 12.3 (L-G) & 0.9 (L-G) & 10.8 \\
\hline & $4-1$ & 103.5 & 549000 & 757000 & 1126294 & 179652 & 1585 & 36.3 (L-G) & $1.6(\mathrm{~L})$ & 29.7 \\
\hline & $6-1$ & 125.5 & 818000 & 661000 & 1306869 & 172854 & 1732 & 34.9 (D) & $2.0(\mathrm{~L})$ & 29.6 \\
\hline & $6-2$ & 104.4 & 479000 & 731000 & 1002682 & 207098 & 1538 & 40.9 (L-G) & $1.6(\mathrm{~L})$ & 32.8 \\
\hline & $8-1$ & 125.6 & 823000 & 685000 & 1335030 & 172867 & 1763 & 34.9 (L-G) & $2.0(\mathrm{~L})$ & 29.6 \\
\hline & $8-2$ & 117.3 & 590000 & 665000 & 1047997 & 206429 & 1757 & 41.7 (L-G) & $2.0(\mathrm{~L})$ & 34.5 \\
\hline & $10-1$ & 131.4 & 909000 & 718000 & 1464370 & 162745 & 1799 & 32.9 (L-G) & $2.0(\mathrm{~L}-\mathrm{G})$ & 28.2 \\
\hline & $10-2$ & 112.8 & 569000 & 750000 & 1120156 & 199249 & 1820 & 40.2 (L-G) & $2.1(\mathrm{~L})$ & 33.8 \\
\hline & $10-3$ & 118.4 & 565000 & 599000 & 947609 & 216119 & 1629 & 43.6 (L-G) & $1.8(\mathrm{~L})$ & 35.3 \\
\hline & $12-1$ & 133 & 905000 & 733000 & 1477203 & 161434 & 1852 & 32.61 & $2.0(\mathrm{~L})$ & 27.9 \\
\hline & $12-2$ & 120.8 & 622000 & 665000 & 1090212 & 197204 & 1731 & 39.8 (L-G) & $1.8(\mathrm{~L})$ & 32.5 \\
\hline & $12-3$ & 117.7 & 574000 & 627000 & 991292 & 209510 & 1658 & 42.3 (L-G) & $1.8(\mathrm{~L})$ & 34.4 \\
\hline \multirow{10}{*}{20} & Z section & 200 & 1823917 & 121500 & 1884701 & 60716 & 933 & 12.3 (L-G) & 0.9 (L-G) & 9.6 \\
\hline & $4-1$ & 106.4 & 572000 & 717000 & 1105919 & 183022 & 1607 & $37.0(\mathrm{~L}-\mathrm{G})$ & & 25.5 \\
\hline & $6-1$ & 128.3 & 862000 & 638000 & 1327752 & 172185 & 1721 & 34.8 (L-G) & 2.0 (L) & 25.8 \\
\hline & $6-2$ & 107.5 & 522000 & 721000 & 1036218 & 206864 & 1640 & 41.8 (L-G) & $1.9(\mathrm{~L})$ & 28.9 \\
\hline & $8-1$ & 129.4 & 866000 & 673000 & 1368055 & 170565 & 1798 & 34.4 (L-G) & $2.0(\mathrm{~L})$ & 25.5 \\
\hline & $8-2$ & 119 & 605000 & 640000 & 1037767 & 206938 & 1738 & 41.8 (L-G) & $2.1(\mathrm{~L})$ & 29.9 \\
\hline & $10-1$ & 132.4 & 904000 & 683000 & 1422108 & 165265 & 1828 & 33.4 (L-G) & $2.0(\mathrm{~L})$ & 25.0 \\
\hline & $10-2$ & 121.7 & 680000 & 635000 & 1117814 & 196935 & 1786 & $39.8(I-G)$ & $2.2(\mathrm{~L}-\mathrm{G})$ & 29.1 \\
\hline & $10-3$ & 121.7 & 625000 & 583000 & 998270 & 209663 & 1635 & 40.2 (D) & 1.9 (L) & 28.3 \\
\hline & $12-1$ & 131.6 & 902000 & 785000 & 1532270 & 155411 & 1816 & $31.4(\mathrm{~L}-\mathrm{G})$ & $2.0(\mathrm{~L}-\mathrm{G})$ & 23.8 \\
\hline
\end{tabular}


$\begin{array}{lllllllll}12-2 & 122.5 & 678000 & 719000 & 1206469 & 191181 & 1877 & 38.6(\mathrm{~L}-\mathrm{G}) & 2.0(\mathrm{~L})\end{array}$

12-3 $120.3 \quad 6470006480001094991200026 \quad 1807 \quad 40.4$ (L-G) 2.2 (L) 29.4

\begin{tabular}{|c|c|c|c|c|}
\hline & 200 & \multicolumn{2}{|c|}{1823917121500188470160716} & 933 \\
\hline
\end{tabular}

4-1 $111.2 \quad 6700007320001227262174440 \quad 1570 \quad 35.2$ (L-G) $\quad 1.7$ (L) 21.5

6-1 $\quad 132.3 \quad 9310006310001396127 \quad 166150 \quad 1711 \quad 33.6(\mathrm{~L}-\mathrm{G}) \quad 1.8(\mathrm{~L}) \quad 21.6$

6-2 $108.85410006960001031925205316 \quad 1655 \quad 41.5$ (L-G) $2.1(\mathrm{~L}-\mathrm{G}) \quad 26.2$

8-1 $133.39490006440001429846163312 \quad 1728 \quad 33.0$ (L-G) $\quad 1.9$ (L) 21.8

8-2 $122.3 \quad 6590006270001085255201384 \quad 1754 \quad 40.7$ (L-G) 2.2 (L-G) 26.1

30

10-1 $\quad 136.99800006460001465675159786 \quad 1797 \quad 32.3$ (L-G) 2.0 (L) 21.6

10-2 $125.87420006180001169191191495 \quad 1795 \quad 38.7$ (L-G) $2.2(\mathrm{~L}-\mathrm{G}) \quad 25.1$

10-3 $122.4 \quad 6660006050001068405202736 \quad 1715 \quad 40.9$ (L-G) 2.2 (L-G) 26.1

12-1 $138.110010007310001577830153545 \quad 1809 \quad 31.0$ (L-G) $\quad 1.9$ (L) 20.9

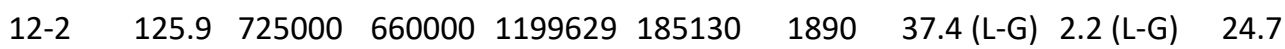

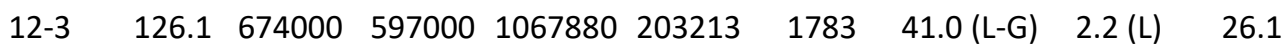

